\title{
Cloud formation in metal-rich atmospheres of hot super-Earths like 55 Cnc e and CoRot7b
}

\author{
G.Mahapatra, ${ }^{1}$ Ch.Helling, ${ }^{1 \star}$ and Y. Miguel $^{2}$ \\ ${ }^{1}$ Centre for Exoplanet Science, SUPA, School of Physics and Astronomy, University of St. Andrews, St. Andrews, UK, KY16 9SS \\ ${ }^{2}$ Laboratoire Lagrange, UMR 7293, Universite de Nice-Sophia Antipolis, CNRS, Observatoire de la Côte d'Azur, \\ Blvd de l'Observatoire, CS 34229, 06304 Nice cedex 4, France
}

Accepted XXX. Received YYY; in original form ZZZ

\begin{abstract}
Clouds form in the atmospheres of planets where they can determine the observable spectra, the albedo and phase curves. Cloud properties are determined by the local thermodynamical and chemical conditions of an atmospheric gas. A retrieval of gas abundances requires a comprehension of the cloud formation mechanisms under varying chemical conditions. With the aim of studying cloud formation in metal rich atmospheres, we explore the possibility of clouds in evaporating exoplanets like CoRoT-7b and $55 \mathrm{Cnc}$ e in comparison to a generic set of solar abundances and the metal-rich gas giant HD149026b. We assess the impact of metal-rich, non-solar element abundances on the gas-phase chemistry, and apply our kinetic, non-equilibrium cloud formation model to study cloud structures and their details. We provide an overview of global cloud properties in terms of material compositions, maximum particle formation rates, and average cloud particle sizes for various sets of rocky element abundances. Our results suggest that the conditions on $55 \mathrm{Cnc}$ e and HD149026b should allow the formation of mineral clouds in their atmosphere. The high temperatures on some hot-rocky superEarths (e.g. the day-side of Corot-7b) result in an ionised atmospheric gas and they prevent gas condensation, making cloud formation unlikely on its day-side.
\end{abstract}

Key words: astrochemistry - Methods: numerical - planets and satellites: atmospheres

\section{INTRODUCTION}

With telescopes of high sensitivity and sophistication in place, we have ventured into an era of observing and characterizing the atmospheres of exoplanets in greater detail. Based on mass and radius measurements, bulk densities are used to draw first conclusions about possible planetary chemical compositions: A rocky bulk composition of an Earth-like silicate/iron mixture appears consistent with masses and radii of short period planets if their radius $<1.5 \mathrm{R}_{\text {Earth }}$ (Dressing et al. 2015). For radii $>1.5 \mathrm{R}_{\text {Earth }}$, bulk densities appear so low that most of these planets must have a large volatile atmosphere (Rogers 2015). The observation and characterization of these atmospheres could provide a unique opportunity to study the internal composition and surface material of rocky exoplanets (Miguel et al. 2011) which is largely unknown. Planetary atmospheres with local gas temperature $>1000 \mathrm{~K}$ have a rich molecular chemistry and they form silicate clouds similar to brown dwarfs (e.g. Sudarsky et al. 2005; Fortney et al. 2008; Witte et al. 2009). Clouds alter the observed spectra by flattening the ultra-violet (UV) and visible spectrum due to scattering from small sized particles, having a cooling effect on the atmosphere beneath the optically

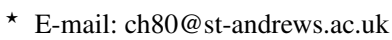

thick region by reflecting the received spectra and also by depleting the local gas-phase of minerals to condense and form mineral cloud particles (Helling \& Casewell 2014).

Even though it is crucial to provide a proper interpretation of observations, cloud formation processes in hot and warm superEarth atmospheres are poorly studied. Clouds have been proposed for super-Earth GJ436 b wherein the observed spectrum in $1.2 \ldots 1.6 \mu \mathrm{m}$ is featureless indicating a possibility of a "heavier than hydrogen" gas-phase molecular composition resulting in high opacity clouds that form at a pressure altitude of $\sim 10^{-3}$ bars (Knutson et al. 2014). Fegley et al. (2016) study the surface out-gassing of hot rocky exoplanets taking into account the fractional vaporization assuming the gas contains the monomers that the rocks are made of. Schaefer et al. (2012) and Schaefer \& Fegley (2009) discuss the possible atmosphere of Corot-7b like planets. They suggest the presence of $\mathrm{Na}$ and $\mathrm{K}$ clouds in the upper parts of the atmosphere. Miguel et al. (2011) explore the composition of initial planetary atmospheres of hot rocky super-Earths (HRSE) in the Kepler planet candidate sample. Ito et al. (2015) conducted a similar study with a few more types of Earth rock compositions. Demory et al. (2016b) have derived a longitudinal temperature map of the super-Earth 55 Cancri-e and find a large temperature contrast of $1300 \mathrm{~K}$ between its day and night sides. Although they suggest the 
temperature map to be a surface map of the planet, Demory et al. (2016b) do not rule out the possibility of a dense atmosphere with thick clouds on the day side.

The aim of this paper is to explore cloud formation in hot and highly enriched exoplanet atmospheres. Atmospheres of small hot planets are poorly understood so that little is known about their composition, chemistry and condensibles. We therefore explore a diversity of scenarios, including extremes. Beside addressing specific objects (CoRoT-7b, $55 \mathrm{Cnc}$ e), this strategy allows to understand our results in a large context by comparing to known and better studied cases (like brown dwarfs which exhibit comparable thermodynamic properties to giant gas planets), and to identify trends regarding cloud properties and the effect of element abundances on cloud properties. We note for clarification that, for example, hydrogen-rich atmospheres for planets like Corot-7b and $55 \mathrm{Cnc}$ e are unlikely unless they are very young as studied by Owen \& Mohanty (2016). Lopez \& Rice (2016)'s Fig. 1 suggest for a planet of $1.58 \mathrm{R}_{\text {Earth }}$ (CoRoT-7b's radius) that a percentage of $\mathrm{H} / \mathrm{He}$ of less than $0.1 \%$ remains in the atmosphere after $5 \mathrm{Gyr}$ for all their highly irradiated cases.

Here we suggest that a rich, non-homogeneous cloud chemistry is to be expected for non-solar and for processed atmospheric element abundances as originating from evaporating planet surfaces or as a result of disk evolution (e.g. Helling et al. 2014; Mordasini et al. 2016; Eistrup et al. 2016a; Cridland et al. 2016) that might lead to locally high metallicities in the gas being accreted by the planets. We explore the evaporating planet CoRoT-7b and the magma-planet $55 \mathrm{Cnc}$ e for various Earth rock compositions as examples for possibly processed, non-solar element abundances. We compare these results with HD149026b for which a 10 times solar metallicity in the element abundances was tested (Fortney et al. 2006a), and use a standard solar element abundance for nonirradiated giant-gas planet atmosphere as reference. We do not consider the physical processes that lead to planetary mass loss which we assume is launched above our computational domain. Intense $\mathrm{X}$-ray and EUV irradiation (driving intense heating and ionisation) in combination with centrifugal acceleration can lead to a hydrodynamic mass loss from the upper atmospheres of hot Jupiters (Khodachenko et al. 2015). Tidal forces will decelerate a wind and MHD effects will confine the wind further to the volume of closed field lines. (Trammell et al. 2011). Due to the complexity of the planetary mass loss models, no consistent link has been made to the underlying atmosphere structure where cloud formation affects the atmosphere structure and determines the observable spectrum, and the inner boundary is not well defined in these complex models. Section 2 describes our approach. We summarise our kinetic cloud formation model and the input properties used. We note that our model neither prescribed the number of seed particles nor the particle size distributions (e.g. Helling \& Fomins 2013). In Sect. 3, we investigate the gas phase composition for different element abundances as pre-requisite for the clouds to form. Section 4 describes the cloud results obtained for different exoplanetary cases (CoRoT7, $55 \mathrm{Cnc}$ e, HD149 026b, reference model) and the influence of different initial element abundances on the cloud structure. Section 5 contains our conclusions.

\section{APPROACH}

We apply our kinetic, non-equilibrium cloud formation model to investigate cloud structures in atmospheres of non-solar element abundances. We assess the impact of element abundances on the gas-phase chemistry and the cloud structure details for prescribed planetary atmosphere profiles.

\subsection{Gas chemistry and kinetic cloud modelling}

Gas-phase chemistry: We apply a chemical equilibrium approach to study the initial, undepleted gas composition for given atmospheric structures $\left(\mathrm{T}_{\text {gas }}, \mathrm{p}_{\mathrm{gas}}\right)$ and the various sets of element abundances described below. We use the code described in Bilger et al. (2013). The cloud formation code contains a chemical equilibrium routine similar to Bilger et al. (2013) but without the large carbohydrate molecules.

Cloud formation model: Our cloud formation model describes the formation of clouds by nucleation, subsequent growth by chemical surface reactions ontop of the seeds, evaporation, gravitational settling, element depletion/enrichment and convective replenishment (Woitke \& Helling 2003, 2004; Helling \& Woitke 2006; Helling et al. 2008b). The effect of nucleation, growth \& evaporation on the remaining elements in the gas phase is fully accounted for (Eqs. 10 in Helling et al. 2008b). The C/O ratio at the start of the cloud formation process is fixed at $\mathrm{C} / \mathrm{O}=0.5$ for all of the initial cloud formation cases so as to be able to discern the differences in the chemistry due to varying silicate compositions. The surface growth of a diversity of materials causes the grains to grow to $\mu \mathrm{m}$-sized particles of a mixed composition. We consider the reactions forming 12 different dust species $\left(\mathrm{TiO}_{2}[\mathrm{~s}], \mathrm{Al}_{2} \mathrm{O}_{3}\right.$ [s], $\mathrm{CaTiO}_{3}$ [s], $\mathrm{Fe}_{2} \mathrm{O}_{3}$ [s], $\mathrm{FeS}[\mathrm{s}], \mathrm{FeO}[\mathrm{s}], \mathrm{Fe}[\mathrm{s}], \mathrm{SiO}$ [s], $\mathrm{SiO}_{2}$ [s], $\mathrm{MgO}[\mathrm{s}], \mathrm{MgSiO}_{3}[\mathrm{~s}], \mathrm{Mg}_{2} \mathrm{SiO}_{4}$ [s]) which react to form grain mantles of a mix of these species depending on the local gas density and temperature. The DRIFT model of Helling \& Woitke (2006) used 60 growth reactions. We treat a total of 79 surface growth reaction in this paper which are provided in Table A2. The seed formation is described by classical nucleation theory which has been modified for $\mathrm{TiO}_{2}$ nucleation to take into account knowledge about $\left(\mathrm{TiO}_{2}\right)_{\mathrm{N}}$ cluster formation (Jeong et al. 1999, 2000; Lee et al. 2015a). A more extensive disucssion of the theoretical background of seed formation is provided in Sect. 3a in Helling \& Fomins (2013). We note that photoionisation may open reaction paths that enable the formation of complex molecular or cluster as precoursers for seed formation at even lower temperatures and densities than considered here (Kirkby et al. 2011; Rimmer et al. 2014). Photochemistry is not part of our present cloud formation model.

\subsection{Input properties}

\subsubsection{Element abundances}

The element abundances are input properties that determine the gas-phase composition of the atmosphere and therefore also the cloud particle material composition. In this study we consider the following species to model exoplanet atmospheres: $\mathrm{H}, \mathrm{He}, \mathrm{Li}, \mathrm{C}, \mathrm{N}$, $\mathrm{O}, \mathrm{Fl}, \mathrm{Ne}, \mathrm{Na}, \mathrm{Mg}, \mathrm{Al}, \mathrm{Si}, \mathrm{S}, \mathrm{Cl}, \mathrm{K}, \mathrm{Ca}, \mathrm{Cr}, \mathrm{Mn}, \mathrm{Fe}, \mathrm{Ni}$, Ti. The undepleted element abundances are called initial abundances $\epsilon_{i}^{0}$. The close-in planets have an extremely hot surface that will melt forming a lava ocean that can vaporise, resulting in an enrichment of the initial composition of the atmosphere. Since the lava ocean composition for an exoplanet is poorly constrained, we adopt different potential compositions for a planetary crust, that will result in different elemental abundances for our enriched atmosphere. The enriched elements are $\mathrm{O}, \mathrm{Mg}, \mathrm{Al}, \mathrm{Si}, \mathrm{Ca}, \mathrm{Ti}, \mathrm{Fe}$, and their initial abundances, $\epsilon_{i}^{0}$, are adopted according to the different compositions for 
Table 1. Initial element abundances, $\epsilon_{i}^{0}$, from magma compositions. Solar and meteorite abundances are shown as a comparison.

\begin{tabular}{ccccccccc}
\hline \hline $\begin{array}{c}\text { Element Abundance } \\
\log \left(\epsilon_{i} / \epsilon_{H}\right)\end{array}$ & Komatiite $^{a}$ & BSE $^{b}$ & BSE $^{c}$ & MORB $^{c}$ & Upper Crust $^{d}$ & Bulk Crust $^{d}$ & Solar $^{e}$ & Meteorite $^{e}$ \\
\hline $\mathrm{Si}$ & 7.54 & 7.54 & 7.54 & 7.54 & 7.54 & 7.54 & 7.54 & 7.55 \\
$\mathrm{O}$ & 8.09 & 8.10 & 8.10 & 7.97 & 8.00 & 7.97 & 8.69 & 8.43 \\
$\mathrm{Mg}$ & 7.52 & 7.62 & 7.64 & 7.01 & 6.61 & 6.29 & 7.54 & 7.56 \\
$\mathrm{Fe}$ & 6.86 & 6.72 & 6.72 & 6.68 & 6.51 & 6.34 & 7.45 & 7.49 \\
$\mathrm{Ca}$ & 6.63 & 6.48 & 6.46 & 6.42 & 6.59 & 6.30 & 6.36 & 6.33 \\
$\mathrm{Al}$ & 6.54 & 6.62 & 6.61 & 6.57 & 7.03 & 6.97 & 6.47 & 6.46 \\
$\mathrm{Na}$ & 5.82 & 5.71 & 5.73 & 5.69 & 6.53 & 6.52 & 6.33 & 6.30 \\
$\mathrm{Ti}$ & 5.12 & 5.01 & 5.06 & 5.66 & 5.49 & 5.39 & 5.02 & 4.95 \\
$\mathrm{~K}$ & 4.93 & 4.56 & 4.47 & 4.79 & 6.12 & 6.27 & 5.08 & 5.11 \\
$\mathrm{Cr}$ & - & - & 5.36 & 4.62 & - & - & 5.64 & 5.67 \\
$\mathrm{P}$ & - & - & 4.12 & 4.77 & 4.79 & 4.82 & 5.36 & 5.44 \\
\hline
\end{tabular}

${ }^{a}$ Schaefer \& Fegley (2004), ${ }^{b}$ O’Neill \& Palme (1998), ${ }^{c}$ McDonough \& s. Sun (1995), ${ }^{d}$ Taylor \& McLennan (2009). ${ }^{e}$ Grevesse et al. (2007).

Earths planetary crust tested in the paper: bulk crust, upper crust, MORB - metal-oxide-ridge basalt, BSE - bulk silicate Earth, komatite (see Table 1 and Sect. A1). All other elements are set to solar values initially, except if all elements are increased by the same factor. Our test cases remain hydrogen rich, unless we specifically test a decreased hydrogen content as in the case 'BSE (low $\mathrm{H}$ )' $\left(10^{-5} n_{H}\right)$. The cloud formation process changes the initial element abundances by nucleation, growth and evaporation with respect to those elements that are involved $(\mathrm{O}, \mathrm{Mg}, \mathrm{Al}, \mathrm{Si}, \mathrm{S}, \mathrm{Ca}, \mathrm{Ti}, \mathrm{Fe}$; see Eqs. 4 \& 8 in Woitke \& Helling (2004)).

Different sets of initial element abundances should influence the local temperature and pressure structure due to changing gas and cloud opacities. Differences in element abundances will also affect the mean molecular weight.

\subsubsection{Atmosphere profiles}

We utilise pre-calculated thermodynamic structure of atmosphere models. The resulting limitation of our approach is therefore that changes of initial element abundances (Sect. 2.2.1) do not affect the $\left(T_{\text {gas }}, p_{\text {gas }}\right)$ structure, nor will the forming cloud particles have an impact on the atmosphere structure considered. We note that the (non-irradiated) Drift-Phoenix giant gas planet model and the models for HD 149026b, that we utilise in this paper, do include the radiative and chemical feedback of clouds on the atmosphere structure. The profile used for $55 \mathrm{Cnc}$ e results from a retrieval approach and should therefore also mimic the presence of clouds in case they are present. We use the following planetary atmosphere profiles for our study of cloud formation in atmospheres with metal enriched abundances:

- non-irradiated giant gas planet as reference and comparison to previous works

(Drift-Phoenix model atmosphere: $T_{\text {eff }}=2500 \mathrm{~K}, \log (\mathrm{g})=3.0$ ),

- the metal-rich mini-giant planet HD $149026 \mathrm{~b}$

$\left(\mathrm{T}_{\text {eff }}=1757 \mathrm{~K}, \log (\mathrm{g})=3.23\right.$; Fortney et al. 2006a $)$,

- CoRoT-7b as hot super-Earth example with $\mathrm{T}_{\text {equ }}=2300 \mathrm{~K}$, $\log (\mathrm{g})=3.62$, adopting the atmosphere model results from Ito et al. (2015) , and

- 55 Cancri e profile as hot-rocky super-Earth example adopting a retrieved profile $\left(\mathrm{T}_{\text {equ }}=2400 \mathrm{~K}, \log (\mathrm{g})=3.33\right.$; Demory et al. 2016a).

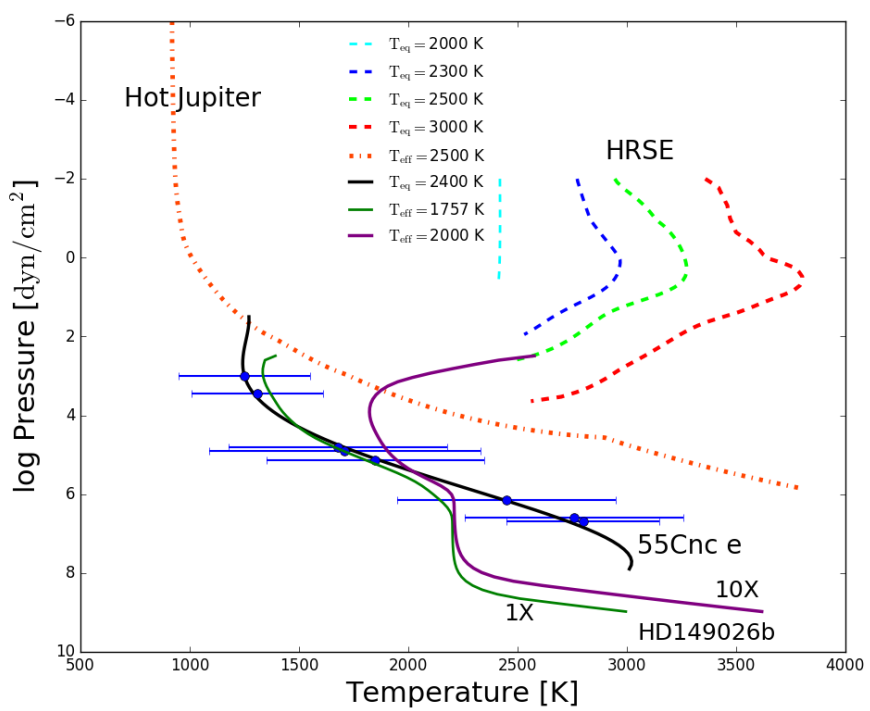

Figure 1. The ( $\left.\mathrm{T}_{\text {gas }}, \mathrm{p}_{\text {gas }}\right)$-profiles of different planetary atmospheres. Drift-Phoenix models with $\mathrm{T}_{\text {eff }}=2500 \mathrm{~K}, \log (\mathrm{g})=3.0$ (orange dashed line; Witte et al. 2009, 2011). Hot rocky super-earths (HRSE) atmosphere profiles are demonstrated for $\mathrm{T}_{\mathrm{eq}}=2000$ to $3000 \mathrm{~K}$ (dashed light blue, blue, green and red lines; Ito et al. 2015). HD149026b with $1 \times$ and $10 \times$ solar element abundances (solid purple and green; Fortney et al. 2006b). $55 \mathrm{Cnc}$ e (solid black and blue points with error bars, Demory et al. 2016a).

Figure 1 compares these different type of atmosphere model structures. Note that different communities use different global temperature definition ${ }^{1}$. The $1 \mathrm{D}\left(\mathrm{T}_{\text {gas }}, \mathrm{p}_{\text {gas }}\right)$ structures for $55 \mathrm{Cnc}$ e, HD

1 The sub-stellar point equilibrium temperature is given as,

$$
\mathrm{T}_{\mathrm{eq}}^{4}=\left(1-\mathrm{A}_{\mathrm{P}}\right) \frac{\mathrm{R}_{*}^{2}}{\mathrm{D}^{2}} \mathrm{~T}_{*}^{4}
$$

where $R_{*}$ and $T_{*}$ are respectively, the radius and temperature of the host star, $A_{P}$ is the planetary albedo, and D is the orbital distance of the planet. The host star is assumed to emit blackbody radiation of $6000 \mathrm{~K}$ and the magma composition is assumed to be BSE. 
Table 2. Global parameters of the planets considered in the paper.

\begin{tabular}{cccccc}
\hline \hline Planets & $\begin{array}{c}\mathrm{a} \\
{[\mathrm{AU}]}\end{array}$ & $\begin{array}{c}\mathrm{T}_{\text {global }} \\
{[\mathrm{K}]}\end{array}$ & $\begin{array}{c}\mathrm{M} \\
{[\mathrm{M} \oplus]}\end{array}$ & $\begin{array}{c}\log (\mathrm{g}) \\
{\left[\mathrm{cm} / \mathrm{s}^{2}\right]}\end{array}$ & References \\
\hline giant gas planet & - & $\mathrm{T}_{\text {eff }}=2500$ & - & 3.0 & Witte et al. (2009) \\
55 Cnc e & $\sim 0.015$ & $\mathrm{~T}_{\text {equ }}=2400$ & 8.63 & 3.33 & Demory et al. (2016a) \\
HD149026b & $\sim 0.042$ & $\mathrm{~T}_{\text {eff }} \sim 1800$ & 114 & 3.23 & Fortney et al. (2006b) \\
CoRoT-7b & $\sim 0.017$ & $\mathrm{~T}_{\text {emu }}=2300$ & $<9$ & $\sim 3.5$ & Ito et al. (2015) \\
\hline
\end{tabular}

$149026 \mathrm{~b}$ and that for the hot non-irradiated giant gas planet for $\mathrm{T}_{\text {eff }}=2500 \mathrm{~K}, \log (\mathrm{g})=3.0$ populate the same part of the $\left(\mathrm{T}_{\text {gas }}, \mathrm{p}_{\mathrm{gas}}\right)$ diagram. The atmospheres profile that was suggested for CoRoT$7 \mathrm{~b}$ by Ito et al. (2015) $\left(\mathrm{T}_{\text {equ }}=2300 \mathrm{~K}\right)$ is far hotter than the gas giant atmosphere profiles. The $\mathrm{T}_{\text {eff }}=2500 \mathrm{~K}$ for the giant-gas planet is chosen since it has a range of local gas temperatures similar to that of hot super Earths or gas-giant planets that we wish to study. CoRoT-7b and 55 Cnc e receive a comparable amount of irradiating flux as they orbit similar stars at a similar distance. The main differences between the two cases are therefore due to the different atmosphere profiles presently available in the literature and potential differences in their atmospheric compositions (55 Cnc e has a larger radius than Corot- $7 \mathrm{~b}$ consistent with a more extended atmosphere). The main differences between We have run our cloud formation model for all these model atmosphere with all element abundances listed in Table 1, but we will later confine our results presentation to a selected set of input atmosphere structures.

Giant gas planet: We use a Drift-PhoenIX model atmosphere with a surface gravity, $\log (\mathrm{g})=3.0$ and effective temperature, $\mathrm{T}_{\text {eff }}=$ $2500 \mathrm{~K}$ for our cloud formation study. Figure 1 shows the atmospheric profiles of two different hot-Jupiters that are derived from the Drift-PhoEnIx model atmosphere simulations described in Witte et al. $(2009,2011)$ for comparison. The atmospheric model used is in local thermal equilibrium (LTE), and the radiative and convective energy transport is solved to determine the local gas temperature. The local gas pressure is calculated assuming hydrostatic equilibrium, and the equations of state, opacity data and equilibrium chemistry calculations close this system of equations; see e.g. (Helling \& Casewell 2014).

Metal-rich mini-giant planets, e.g. HD 149 026b: HD 149026b is an extrasolar giant planet with an orbit of 2.87 days around a metal-rich G0IV parent star. It has a radius of only $0.725 \mathrm{R}_{J} \pm$ $0.05 \mathrm{R}_{J}$ and a mass of $0.36 \mathrm{M}_{J} \pm 0.03 \mathrm{M}_{J}\left(114 \mathrm{M}_{\oplus}\right.$, Sato et al. 2005). Evolution models suggest that the planet should have larger radii (Guillot et al. 1996), but it is decidedly small. Fortney et al. (2006b) provide atmosphere models for the planet and suggest that a hot stratosphere (temperature inversion) may develop due to additional flux absorption by $\mathrm{TiO}$ and $\mathrm{VO}$. The $\left(\mathrm{T}_{\mathrm{gas}}, \mathrm{p}_{\mathrm{gas}}\right)$ profiles used in our study are shown in Fig. 1, where $1 \times$ (green) corresponds to solar abundance and $10 \times$ (purple) corresponds to a 10 times solar atmospheric profile. This model allows us to study the effect of an increase of element abundances by the same amount for each element compared to the solar element abundances.

Hot Super-Earth CoRoT-7b (HRSE): We use ( $\left.\mathrm{T}_{\text {gas }}, \mathrm{p}_{\text {gas }}\right)$ profiles from Ito et al. (2015) for HRSE for a first assessment of cloud formation and their potential details for CoRoT-7b should cloud formation be possible. The atmosphere simulation by Ito et al. (2015) is a 1D plane-parallel thermal structure which is in radiative, hydrostatic and chemical equilibrium. These models use the ground pressure, $P_{g}$ and molar fraction $x_{A}$ which are the functions of the ground temperature $T_{g}$ which is determined assuming the magma ocean is a blackbody. No clouds are considered in these models. Figure 1 shows several HRSE ( $\mathrm{T}_{\text {gas }}, \mathrm{p}_{\mathrm{gas}}$ )-profiles provided by Ito et al. (2015) for hot super-Earths with $\mathrm{T}_{\mathrm{eq}}$ from 2000 to $3000 \mathrm{~K}$. In the cases of $T_{\mathrm{eq}} \leqslant 2000 \mathrm{~K}$, the atmosphere is isothermal because the atmosphere is so optically thin that the ground is directly heated by the stellar irradiation (Ito et al. 2015). Ito et al. (2015) used $\mathrm{T}_{\text {equ }}=2300 \mathrm{~K}$ to represent CoRoT- $7 \mathrm{~b}$.

Possible atmosphere on 55 Cnc e: 55 Cnc e is an interesting candidate to study the potential atmospheric compositions due to its mass and radius estimated at $8.09 \pm 0.26 \mathrm{M}_{\oplus}$ (Nelson et al. 2014) and $2.17 \pm 0.10 \mathrm{R}_{\oplus}$ (Gillon et al. 2012) which makes it fall into the category of a Super-Earth. Given the extremely high equilibrium temperature $\left(\mathrm{T}_{\mathrm{eq}} \sim 2400 \mathrm{~K}\right)$ and its proximity to the parent star, it is highly likely that the planetary lithosphere is weak and most of the day-side surface of the planet will be in a semi-molten or molten state which would lead to magma oceans and possibly volcanic activity on the irradiated day-side (Demory et al. 2016a).

Due to the high brightness of the host star, $55 \mathrm{Cnc}$ e can be observed with great detail in the visible as well as Spitzer $4.5 \mu \mathrm{m}$ IRAC Photometric band (Demory et al. 2012; Winn et al. 2011; Gillon et al. 2012). Based on the precise measurement of mass and radius alone, Demory et al. (2011) suggested a silicate-rich interior with a dense $\mathrm{H}_{2} \mathrm{O}$ envelope of $20 \%$ by mass, Gillon et al. (2012) suggested a purely silicate planet with no envelope and Madhusudhan (2012) suggested a carbon-rich planet with no envelope, Demory et al. (2016a) suggested an atmospheric model in which multiple volcanic plumes explain the large observed temperature variations on the day-side. The $\left(\mathrm{T}_{\text {gas }}, \mathrm{p}_{\text {gas }}\right)$ profile that we use in our study is derived from the figure in Demory et al. (2016a). This profile was retrieved based on the observed IRAC $4.5-\mu \mathrm{m}$ brightness temperature $\left(\mathrm{T}_{B}\right)$ between $\mathrm{T}_{\min }=1273_{+271 K}^{-348 K}$ and $\mathrm{T}_{\max }=2816_{+358 K}^{-368 K}$. Such retrieved planetary atmosphere are therefore isothermal at the upper and the lower boundary, in contrast to complete forward models like Drift-Phoenix or those from Ito et al. (2015) and Fortney et al. (2006b).

\subsection{Atmospheric mixing}

The vertical mixing is parametrized using the eddy diffusion coefficient $\mathrm{K}_{z z}$ which represents the strength with which material can be transported into higher atmospheric layers (Agúndez et al. 2014). There has been extensive research on the choice of $\mathrm{K}_{z z}$ for the cases of hot-Jupiters (Moses et al. 2011; Miguel \& Kaltenegger 2013; Parmentier et al. 2013; Agúndez et al. 2014). Parmentier et al. (2013) use vertical diffusive coefficients determined by following passive tracers in their general circulation model (GCM). They adopt a $\mathrm{K}_{z z}\left[\mathrm{~cm}^{2} \mathrm{~s}^{-1}\right]=5 \times 10^{8} \mathrm{p}^{-0.5}$ bar for HD $209458 \mathrm{~b}$ which is a gas-giant. Agúndez et al. (2014) used $\mathrm{K}_{z z}\left[\mathrm{~cm}^{2} \mathrm{~s}^{-1}\right]=10^{7}$ $\mathrm{p}^{-0.65}$ (bar) for the case of HD $189733 \mathrm{~b}$ and Miguel \& Kaltenegger (2013) considered values between $\mathrm{K}_{z z}\left[\mathrm{~cm}^{2} \mathrm{~s}^{-1}\right]=10^{8} \ldots 10^{9}$ for their atmospheric models of mini-Neptunes. We follow the approach detailed in Lee et al. (2015a) for the vertical mixing. The diffusion mixing time scale is derived from $\tau_{\text {mix }}=$ const $\cdot \mathrm{H}_{\mathrm{p}}^{2} / \mathrm{K}_{\mathrm{zz}}$ for a prescribed diffusion constant $\mathrm{K}_{z z}$. A constant $\mathrm{K}_{z z}=10^{11} \mathrm{~cm}^{2}$ $\mathrm{s}^{-1}$ was chosen for $55 \mathrm{Cnc}$ e. Tests have shown that for the $55 \mathrm{Cnc}$ e profile (black line joining blue dots with error bars in Fig. 1), cloud formation does not start for $\mathrm{K}_{z z}<10^{9} \mathrm{~cm}^{2} \mathrm{~s}^{-1}$. The $\mathrm{K}_{z z}$ value for HD $149026 \mathrm{~b}$ is selected to be $10^{13} \mathrm{~cm}^{2} \mathrm{~s}^{-1}$. For CoRoT-7b, $\mathrm{K}_{z z}$ was varied between values ranging from $10^{7} \mathrm{~cm}^{2} \mathrm{~s}^{-1}$ to $10^{15} \mathrm{~cm}^{2} \mathrm{~s}^{-1}$. 

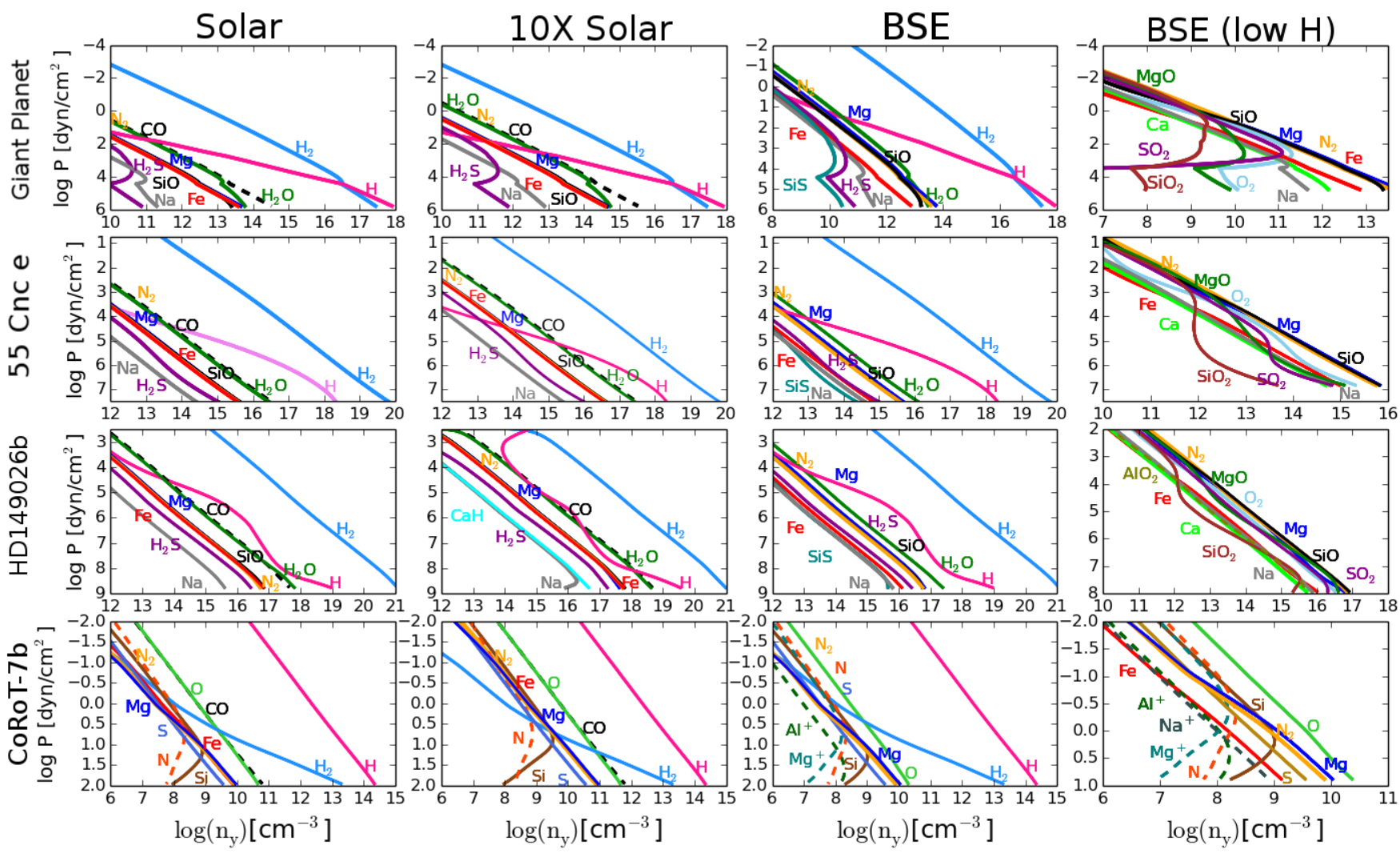

Figure 2. The gas-phase number densities, $\mathrm{n}_{\mathrm{y}}\left[\mathrm{cm}^{-3}\right]$, of the 10 most abundant gas species for four types of elements abundances and four different $\left(\mathrm{T}_{\text {gas }}\right.$, $p_{\text {gas }}$ )-profiles. Different rows represent different atmospheric models as indicated and the respective $\left(T_{\text {gas }}, p_{\text {gas }}\right)$-profiles are shown in Fig. 1 . The name of each gas species is indicated near the line with the same color. Different columns represent different sets of initial element abundances: solar, $10 \times$ solar $-10 \times \epsilon_{\text {solar }}$, BSE(low H) - BSE abundance with $10^{-5} \times \epsilon_{\mathrm{H}}$

\section{RESULTS: EQUILIBRIUM GAS-PHASE COMPOSITION}

Clouds form from an atmospheric gas if the appropriate thermodynamic conditions are met. Their composition depends on the chemical composition of the atmospheric gas. Witte et al. (2009) demonstrated how a (homogeneously) decreasing element abundance changes the cloud structure in brown dwarf atmospheres by reducing the set of solar element abundances by the same factor from $[\mathrm{M} / \mathrm{H}]=0.0 \ldots-6.0$. Recent chemical modelling efforts in planet forming disks show that planets will most likely form from a set of initial element abundances that is non-solar nor a simple scaling of the solar set of element abundances (Helling et al. 2014; Eistrup et al. 2016b). In this section we show the results and analyze the chemical gas-phase composition for all the cases explored in this paper. To keep the number of panels sensible, we chose 4 sets of element abundances only (solar, 10x solar, BSE and BSE with a low $\mathrm{H}$ abundance). We note that all figure will also contain results for extreme cases for completeness and for reference. These extreme cases are the hydrogen-rich atmospheres for $55 \mathrm{Cnc}$ e and CoRoT-7b which may only be realistic during a very short time of their infancy or when kicked back into a hydrogen-rich part of the planet-forming disk. The two BSE-cases for the giant gas planet and HD $149026 \mathrm{~b}$ can represent stages of a heavy influx of meteorits or even planetary collisions.

Figure 2 shows the 10 most abundant species that result from our chemical equilibrium gas-phase calculations. In Figures 3 to 5 we show the results for the chemistry of one element at a time (Fig. 3: Si, Fig. 4: $\mathrm{Mg}$ and Fe, Fig. 5: Al and Ti). We generally observe that a lower hydrogen content (here: BSE (low $\mathrm{H}$ )) leads to higher abundances of oxides such as $\mathrm{SiO}_{2}, \mathrm{TiO}_{2}, \mathrm{MgO}, \mathrm{FeO}, \mathrm{AlO}_{2} \mathrm{H}$.

Giant gas planet: The atmosphere is dominated by volatiles for the solar, metal enriched solar (10x solar) and BSE cases. In the case of solar abundance, the atmosphere is primarily composed of $\mathrm{H}_{2}, \mathrm{H}, \mathrm{CO}$, as shown in Figure 2. Increasing the element abundances homogeneously results in an increase of the molecular number densities (compare 1st and 2nd column in Figs. 3 - 5). This effect is stronger for $\mathrm{Mg}, \mathrm{Si}$ and $\mathrm{Fe}$ than for elements with an intrinsically lower abundance like $\mathrm{Ti}$ and $\mathrm{Al}$.

The BSE atmosphere has an increase in $\mathrm{H}_{2} \mathrm{O}, \mathrm{SiO}, \mathrm{SiS}, \mathrm{K}, \mathrm{Al}$, TiO among others (see Figure 2). The differences in the solar and $\mathrm{BSE}$ abundances with a fixed $\mathrm{C} / \mathrm{O}$ ratio of 0.5 is quite pronounced in the higher pressure regions where the amount of $\mathrm{TiO}$ and $\mathrm{TiO}_{2}$ increases slightly with a drastic decrease in $\mathrm{TiC}$ molecules in the BSE case. An increase in such UV absorbers may therefore induce a local temperature inversions (Fortney et al. 2006a). The strongest changes with metallicity occurs for the Mg-binding species.

55Cnc e: The atomic species dominate the gas-phase for all elements in the case of BSE composition. Tsiaras et al. (2015) in their analysis of an atmosphere around $55 \mathrm{Cnc}$ e, show that the abundances of $\mathrm{HCN}$ and $\mathrm{C}_{2} \mathrm{H}_{2}$ increases while the abundance of $\mathrm{H}_{2} \mathrm{O}$ decreases drastically which is similar to the results that we obtain 

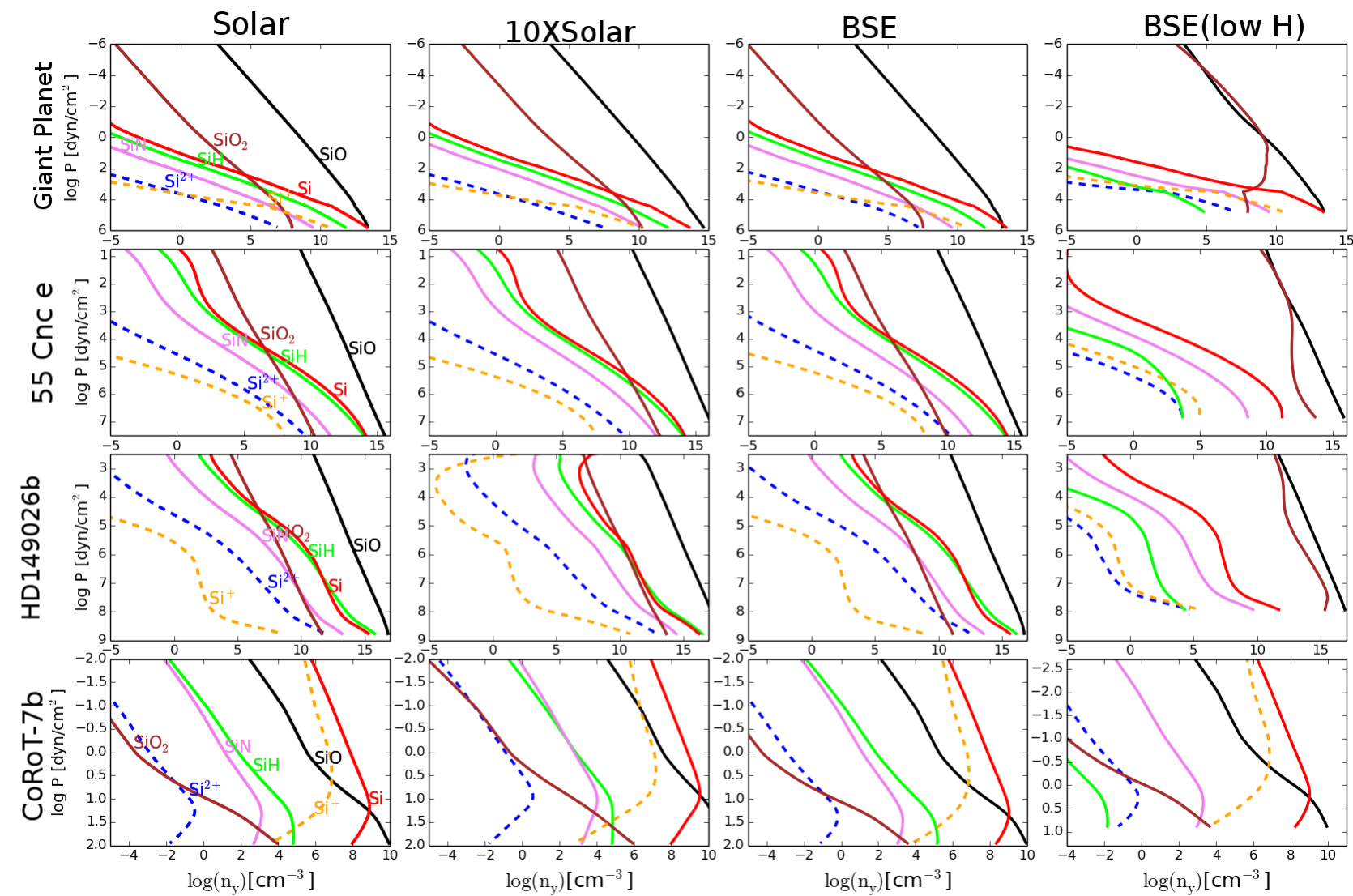

Figure 3. The gas-phase number densities, $\mathrm{n}_{y}\left[\mathrm{~cm}^{-3}\right]$, for Si-binding species in chemical equilibrium for four types of elements abundances and four different ( $\mathrm{T}_{\text {gas }}, \mathrm{p}_{\text {gas }}$ )-profiles. Different rows represent different atmospheric models as indicated and the respective ( $\mathrm{T}_{\text {gas }}, \mathrm{p}_{\text {gas }}$ )-profiles are shown in Fig. 1. Different columns represent different sets of element abundances: 10×solar $-10 \times \epsilon_{\text {solar }}$, BSE $\left(\right.$ low H) - BSE abundance with $10^{-5} \times \epsilon_{\mathrm{H}}$. All species have the same line color in all panels and are provided in column 1.

for a test $\mathrm{BSE}$ composition with $\mathrm{C} / \mathrm{O}=1.0$ (not shown) as compared to a oxygen-rich solar abundance.

Metal-rich mini-giant planets, e.g. HD149026b: The third rows of Figs. 3, 4 and 5 show the gas-phase composition for HD149026b. The temperature inversion is seen at around $2700 \mathrm{~K}$ where all the gases go through a sudden increase in concentration. The second panel of the third row for the figures show the plots for an enriched atmosphere with 10x the solar metallicity. The abundance of oxides of $\mathrm{Ti}, \mathrm{Al}, \mathrm{Fe}, \mathrm{Mg}$ and $\mathrm{Si}$ is higher for HD149026b as compared to other planets which would add to the cloud opacity by imposing strong cloud particle growth.

Hot Super-Earth CoRot 7b: The fourth row of Figs. $3-5$ show the number densities of the dominating species for CoRoT-7b case. The local gas temperatures for this planet are considerably higher compared to the other three planetary model atmospheres due to the stronger irradiation and the hight temperatures profiles adopted from ?. The atmospheric model of CoRoT-7b is representative of a rocky planet. Due to the closeness to its host star and high irradiation, the volatiles might have escaped. Therefore we would expect the BSE case with a low hydrogen content to be the most representative of CoRoT-7b initial element abundances. A comparison between BSE case with the solar element abundances shows the sensitivity of the gas composition to uncertainties in initial element abundances. We note that a changing hydrogen-content will affect the mean molecular weight of the whole atmosphere.

The atmosphere of CoRoT-7b is primarily composed of gases in their atomic and ionic states, hydrogen is present as $\mathrm{H}$ (see Fig. 2). Si species such as $\mathrm{SiO}$ and $\mathrm{SiS}$ are still quite dominant. We find most of the other elements such as $\mathrm{Ti}, \mathrm{Mg}, \mathrm{Fe}$ and $\mathrm{Al}$ to be dominant in their atomic and ionic states in both solar and BSE atmospheres. $\mathrm{SiO}$ dominates the Si-binding gas species at high gas pressures, followed by $\mathrm{Si}$ and $\mathrm{Si}^{+}$. This hierarchy is somewhat metallicity dependent. $\mathrm{Mg}, \mathrm{Fe}, \mathrm{Al}$ and $\mathrm{Ti}$ appear always in their atomic states followed by their singly ionised state $\left(\mathrm{Mg} / \mathrm{Mg}^{+}\right.$, $\left.\mathrm{Fe} / \mathrm{Fe}^{+}, \mathrm{Al} / \mathrm{Al}^{+}, \mathrm{Ti} / \mathrm{Ti}^{+}\right) . \mathrm{Al}^{+}$and $\mathrm{Ti}^{+}$appear at lower pressures than $\mathrm{Mg}^{+}$or Fe${ }^{+}$for the CoRoT-7b profile utilised here. Therefore, the atmosphere of CoRoT-7b is strongly ionised on its day-side. This suggests that either no cloud formation can take place or other cloud formation paths than those considered here need to be considered. For example, noctilucent clouds on Earth are suggested to form through ion-cluster nucleation.

A decreasing hydrogen abundance does not affect these results significantly with respect to the most abundant species of each element.

\subsection{Summary}

We have analyzed the gas-phase composition for different set of initial abundances as starting point for cloud formation. The pri- 

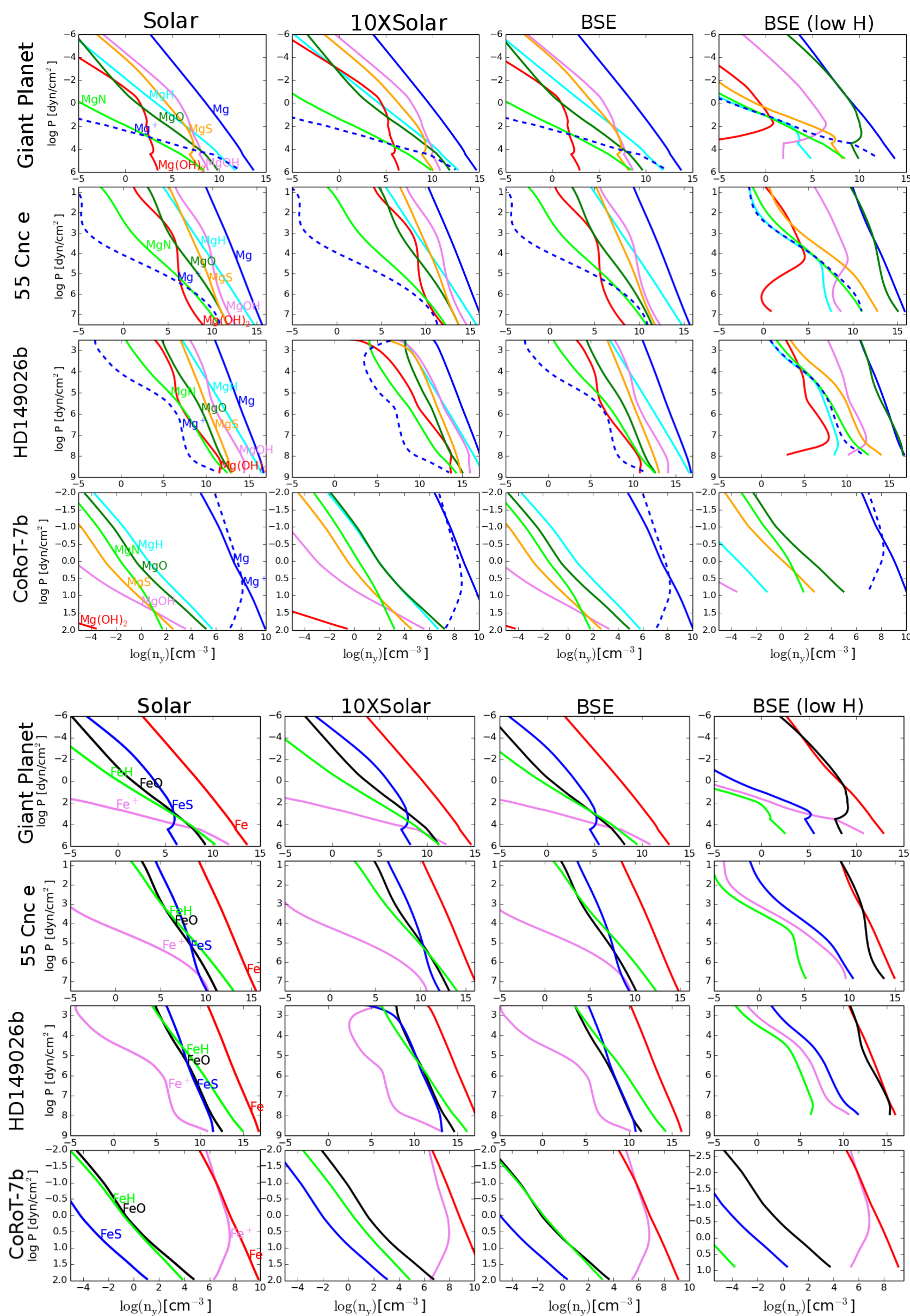

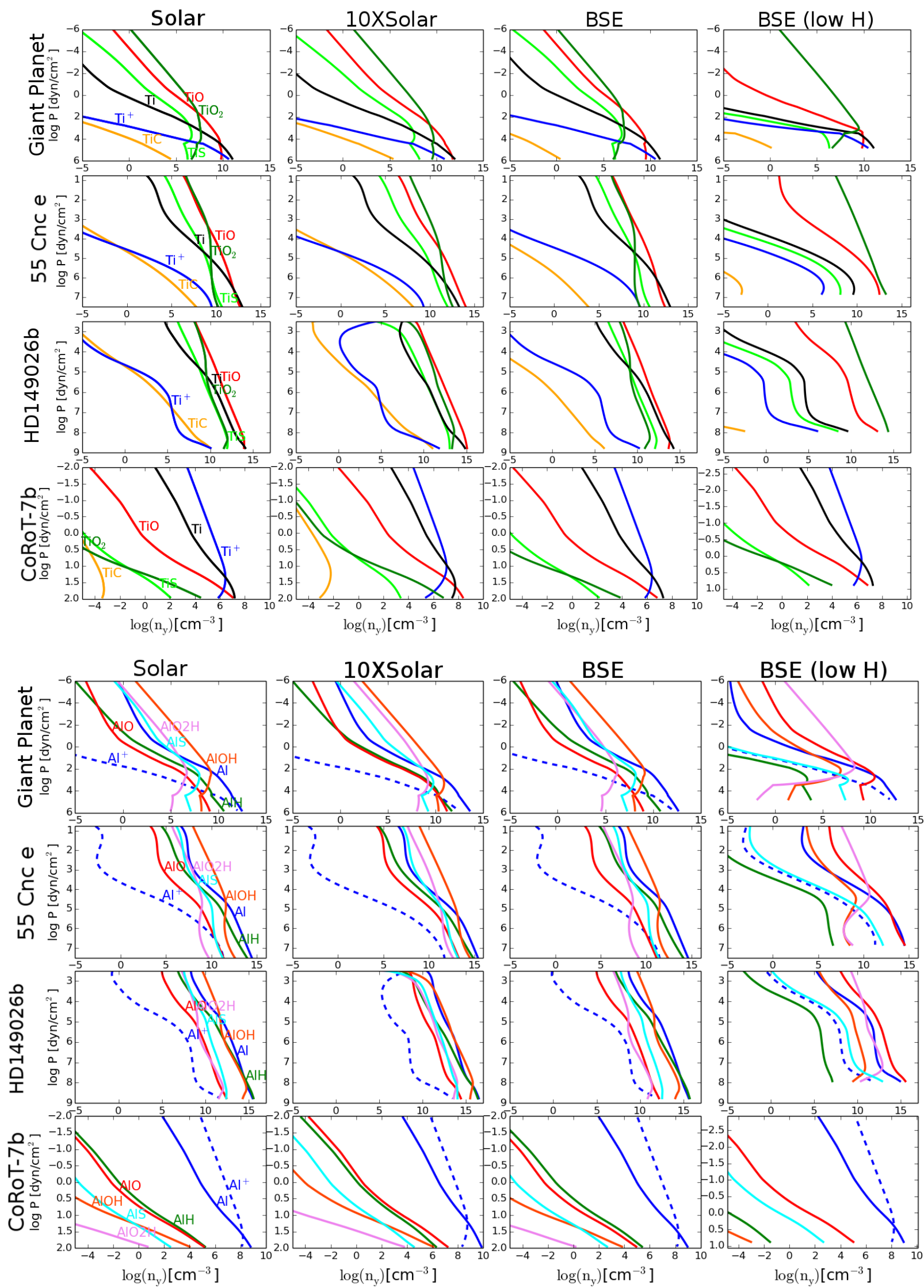

Figure 5. Same like Fig. 3 but for Ti-binding (top) and Al-binding (bottom) gas-phase species. 


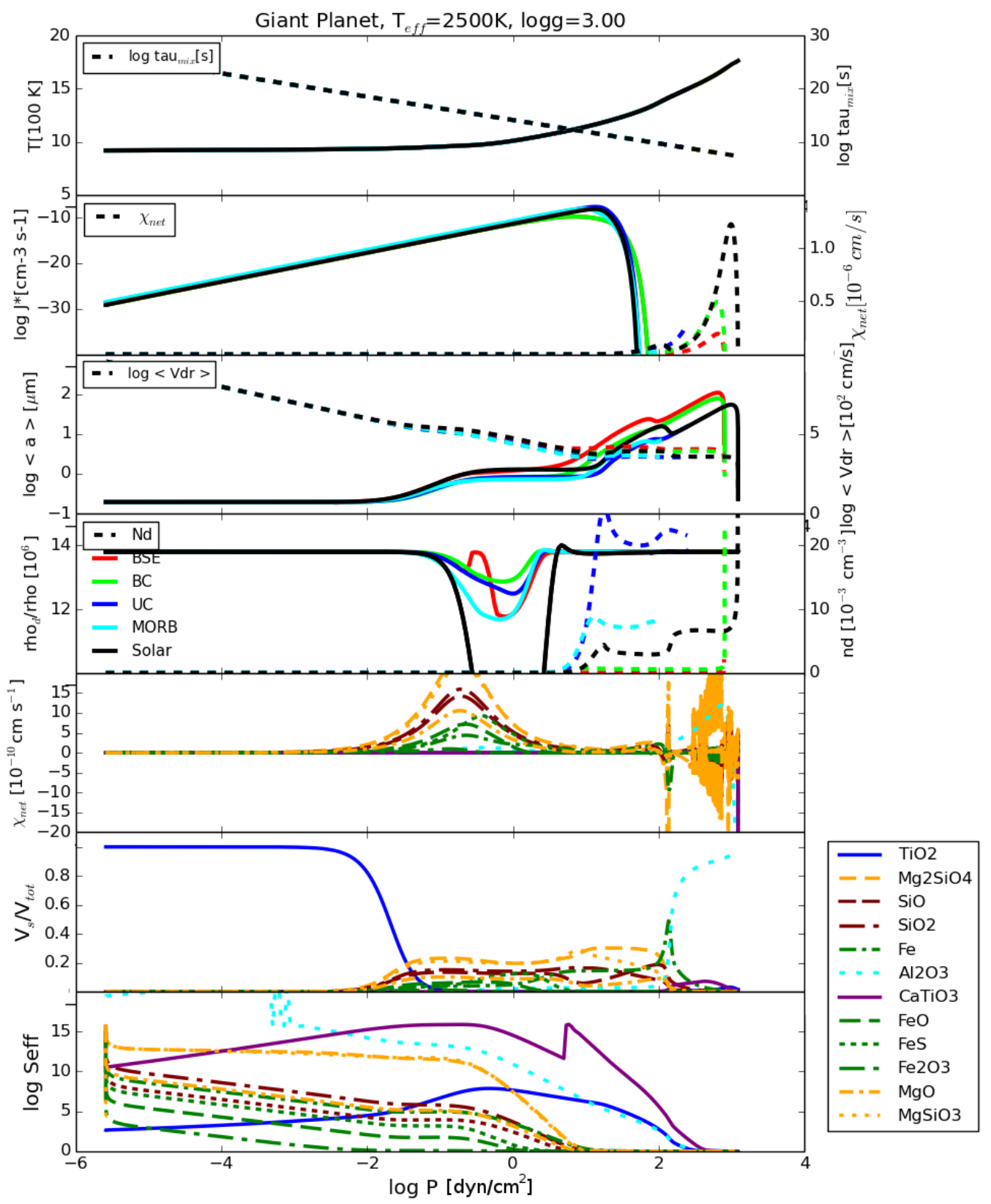

Figure 6. Cloud structure and cloud details for a giant gas planet $\left(\mathrm{T}_{\text {eff }}=2500 \mathrm{~K}, \operatorname{logg}=3.0\right)$ DrIFT-PhoENIX atmosphere model. 1st panel: local gas temperature, $\mathrm{T}_{\text {gas }}$ (solid), mixing time scale $\tau_{\text {mix }}$ (dashed); 2nd panel: Nucleation rate, $\log J_{*}$ (solid), dust growth velocity, $\chi_{\text {net }}$; 3rd panel: mean cloud particle size, $\log \langle a\rangle$, drift velocity, $\log \left\langle v_{\mathrm{dr}}\right\rangle$; 4th panel: dust-to-gas mass ratio, $\rho_{\mathrm{d}} / \rho_{\text {gas }}$, particle number density, $\mathrm{n}_{\mathrm{d}}$. The cloud properties based on five sets of initial element abundances (solar - black, BSE - red, Bulk Crust - green, Upper Crust - blue, MORB - sky blue) are shown in different colors in panels 2, 3, and 4. Panels 5 and 6 are for solar element abundances only, and the results are detailed for the 12 different growth species $s$ : 5th panel: grain growth velocity, $\chi_{\mathrm{s}}$; 6th panel: material volume fraction, $\mathrm{V}_{\mathrm{s}} / \mathrm{V}_{\text {tot }} ;$ 7th panel: effective supersaturation ratio, $\log S_{\text {eff }}$.

mary abundant species is $\mathrm{H}_{2}$ in all of our atmosphere's except that of CoRoT-7b which has atomic $\mathrm{H}$ as the dominating species due to its higher gas temperatures. The BSE atmosphere model used for CoRoT-7b suggests the presence of gases such as $\mathrm{SiO}, \mathrm{Mg}, \mathrm{K}$, $\mathrm{Na}, \mathrm{O}$ which are also found in the atmospheres of $55 \mathrm{Cnc}$ e and HD149026b. SiO is the dominant gas resulting from $\mathrm{Si}$ in all of the planet cases except CoRoT-7b where atomic $\mathrm{Si}$ is the dominant gas-phase species due to higher local gas temperatures (except at high gas pressures). It is followed by $\mathrm{SiO}_{2}$ in the upper parts of the atmospheres for the gas giant, $55 \mathrm{Cnc}$ e and HD149026b.
Atomic $\mathrm{Fe}$ is the dominant species among the Fe-binding gasphase species, for all of the planets and element compositions considered here. Similarly, atomic $\mathrm{Mg}$ is the dominant species among all of the Mg bearing gas-phase molecules, except for HD149026b with BSE (low $\mathrm{H}$ ) where we see $\mathrm{MgO}$ as another dominant species. However, giant planets with low hydrogen content are an extreme and most likely unrealistic case. Our results suggest that the chemical gas-phase composition does not change drastically if the element abundances are increased by the same factor for all elements starting from solar composition (up-scaled solar composition), but 

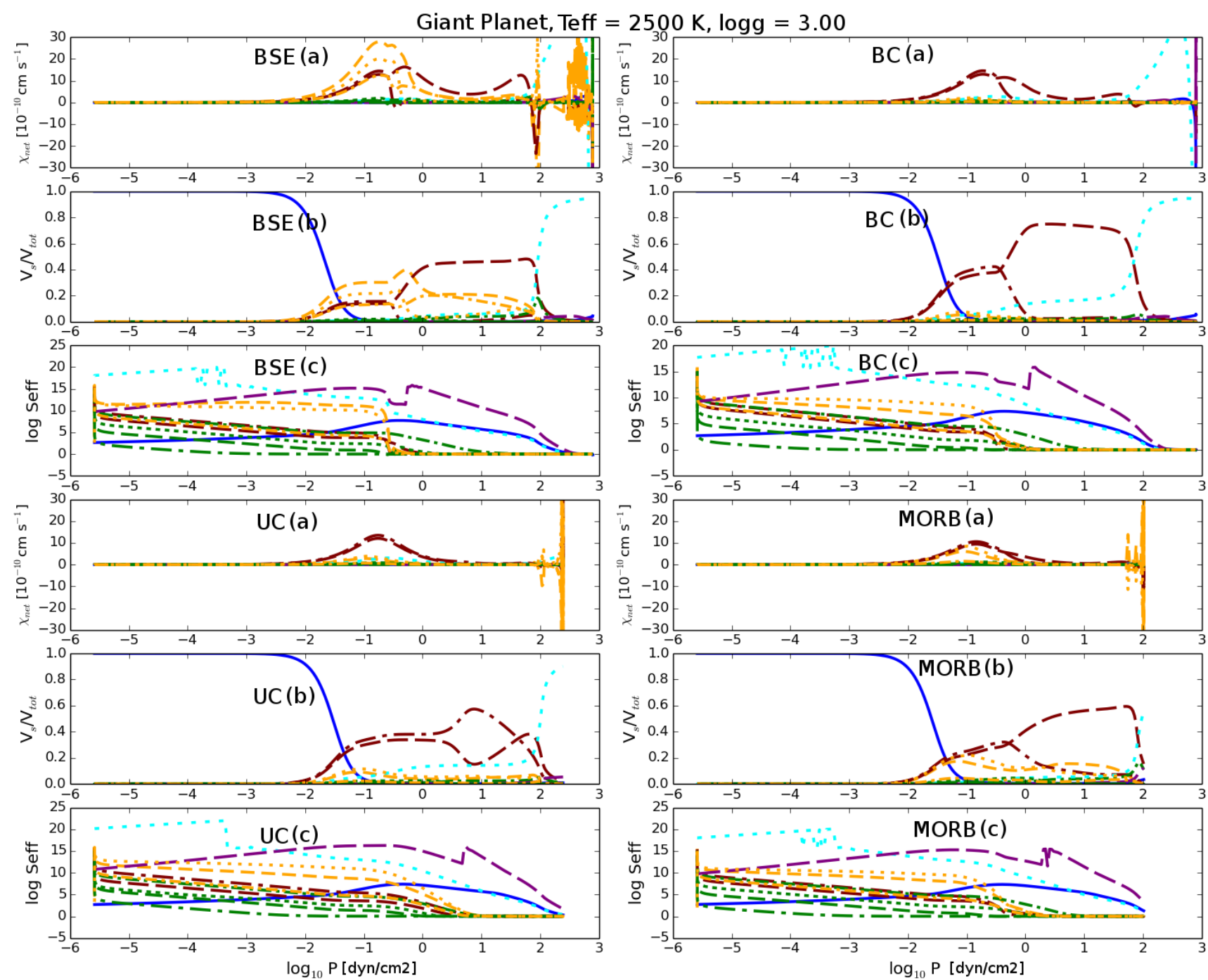

$\begin{aligned}- & \mathrm{TiO} 2 \\ -- & \mathrm{Mg} 2 \mathrm{SiO} 4 \\ -- & \mathrm{SiO} \\ -- & \mathrm{SiO} 2 \\ --- & \mathrm{Fe} \\ -- & \mathrm{Al} 2 \mathrm{O} 3 \\ - & \mathrm{CaTiO} 3 \\ -- & \mathrm{FeO} \\ -- & \mathrm{FeS} \\ - & \mathrm{Fe} 2 \mathrm{O} 3 \\ -- & \mathrm{MgO} \\ \ldots- & \mathrm{MgSiO} 3\end{aligned}$

Figure 7. The cloud particle properties results for a giant gas planet $\left(\mathrm{T}_{\mathrm{eff}}=2500 \mathrm{~K}, \operatorname{logg}=3.0\right)$ DRIFT-PновNIx atmosphere model for four different sets of initial element abundances in detail for the 12 different growth species $s$ : (a) grain growth velocity, $\chi_{\mathrm{s}}\left[\mathrm{cm} \mathrm{s}^{-1}\right]$; (b) material volume fraction, $\mathrm{V}_{\mathrm{s}} / \mathrm{V}_{\text {tot }}[\%] ;(\mathbf{c})$ effective supersaturation ratio, $\log S_{\text {eff. }}$ The four compositions BSE, BC, UC, MORB have been labeled on each plot.

it leads to higher gas-phase concentrations of enriched elements (as can be seen in the second column of Figs. 3, 4, 5). We note that our gas-phase results for CoRot-7b so far suggest that the atmosphere is highly ionised and plasma process may therefore effect the atmosphere (Helling et al. 2016a).

\section{CLOUDS IN ATMOSPHERES OF NON-SOLAR ELEMENT ABUNDANCES}

Table 2 summarizes the global parameters of the planets that we use to evaluate cloud structures for planets that have been suggested to possess potentially non-solar sets of initial element abundances.

Figures 6,8 and 10 show the input $\left(\mathrm{T}_{\text {gas }}, \mathrm{p}_{\text {gas }}, \tau_{\text {mix }}\right)$ in the top panel. Panel 2-4 demonstrate governing cloud variable (nucleation rate $J_{*}\left[\mathrm{~cm}^{-3} \mathrm{~s}^{-1}\right]$, net growth velocity $\chi_{\text {net }}[\mathrm{cm} / \mathrm{s}]$, equilibrium drift velocity $\mathrm{v}_{\mathrm{dr}}[\mathrm{cm} / \mathrm{s}]$ ) and resulting cloud properties (cloud number density $\mathrm{n}_{\mathrm{d}}\left[\mathrm{cm}^{-3}\right]$, mean cloud particle size $\langle a\rangle[\mu \mathrm{m}]$, dust-to-gas ration $\rho_{\mathrm{d}} / \rho_{\text {gas }}$ ) for 5 sets of element abundances (BSE, BC, UC,
MORB, solar). Panels 5, 6, and 7 present the results for the set of solar element abundances only but now detailed for the 12 individual growth materials involved (see Sect. 2.1): net growth velocity $\chi_{\text {net }}^{\mathrm{s}}[\mathrm{cm} / \mathrm{s}]$ for material $s$, the volume fraction $V_{\mathrm{s}} / V_{\text {tot }}$ for material $s$ and the effective supersaturation ration $\mathrm{S}_{\text {eff }}$ for each material $s$. Figures 7, 9 and 11 contain 4 groups of three panels that are similar to Panels 5, 6, and 7 in Figs. 6, 8 and 10 but for the remaining sets of element abundances (BSE, UC, BC, MORB).

\subsection{Clouds that form in the atmosphere of a giant gas planet}

Figure 6 shows how the cloud properties and, hence, the cloud structure changes in the case of different sets of initial element abundances for a sample atmosphere representative of a nonirradiated gas-giant $\left(\mathrm{T}_{\mathrm{eff}}=2500 \mathrm{~K}, \log (\mathrm{g})=3.0\right)$ being representative for a long-period or free-floating planet.

The nucleation rates $\left(\mathrm{J}_{*}\right.$, panel 2 , left) appear similar for the sets of element abundances, however bulk-crust (BC) abundance causes a less efficient nucleation at the nucleation peak. BC fur- 


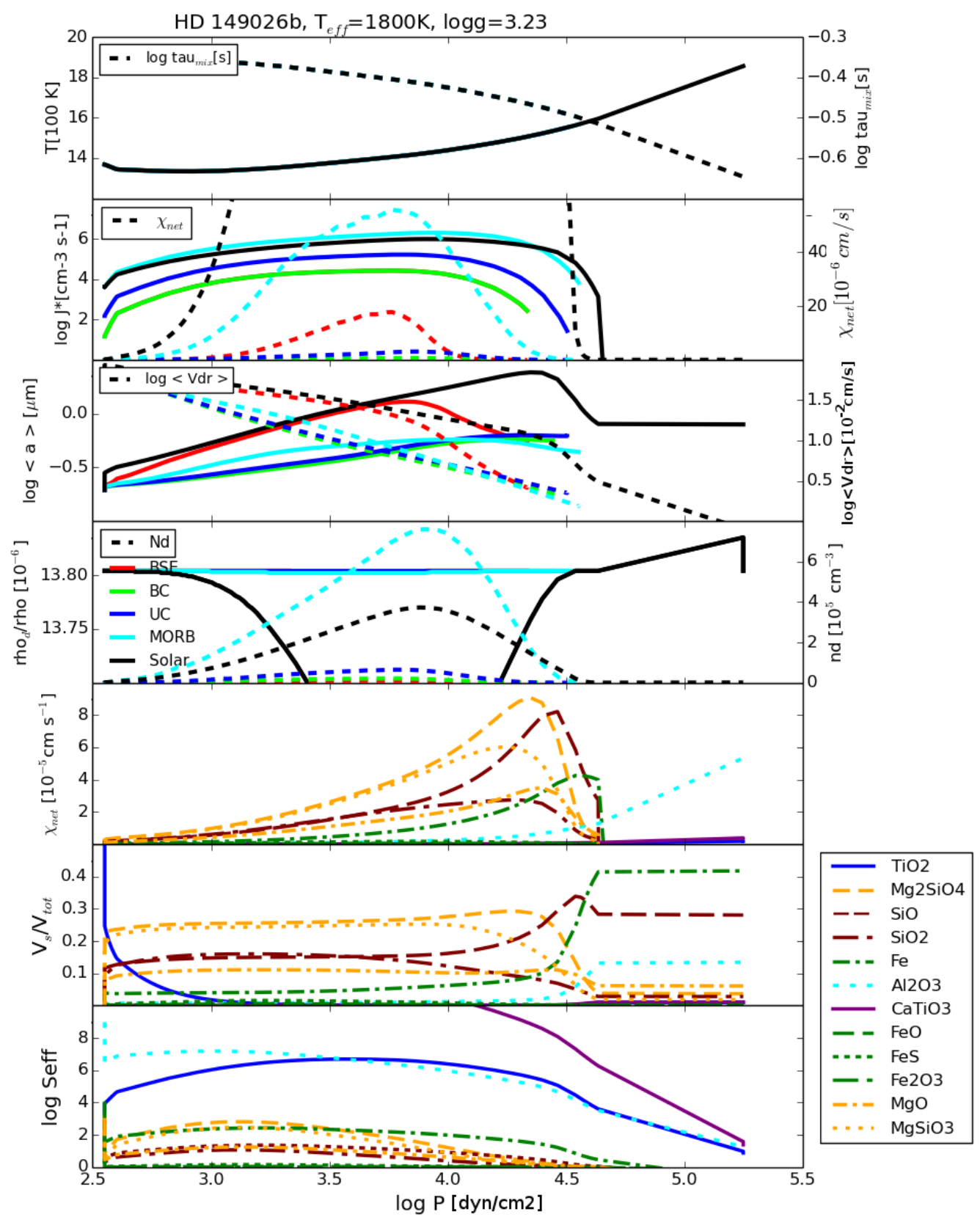

Figure 8. Cloud structure and cloud details for HD149026b $\left(\mathrm{T}_{\mathrm{eff}}=1757 \mathrm{~K}, \operatorname{logg}=3.23\right)$ for five sets of initial element abundances (panel 2, 3, 4). The figure has the same structure like Fig. 6.

ther allows the nucleation to spread somewhat more towards higher temperatures and higher gas pressure than any of the other sets of element abundances. The net growth velocity $\left(\chi_{\text {net }}\right.$, panel 2 , right) reaches largest values for solar abundance. There is significant variation in the final particle sizes $(\langle a\rangle$, panel 3, left) before the cloud particles evaporate. The particles for the BSE set of initial element abundances reach the biggest sizes followed by the BC, solar and then MORB and upper-crust (UC) abundance sets. This is a clear hierarchy of available gas-phase growth species rather than an effect of the local gas temperature because no detailed feedback onto the gas temperature is taken into account in our present work based on the new cloud structures derived here. The underlaying model atmospheres from Drift-PhoENIX and from Fortney et al. (2006b) do include the effect of clouds on the temperature structure and el- ement abundances. This is also seen from the drift velocities ( $v_{d r}$, panel 3, right) with BSE particles being the biggest and having the largest drift velocity. The individual material volume fractions $\left(V_{\mathrm{s}} / V_{\mathrm{tot}}\right.$, panel 6) reflect the individual net growth velocities $\left(\chi_{\mathrm{net}}^{\mathrm{s}}\right.$, panel 5) of each of the materials, $s$, and demonstrates where growth is most efficient for which material. The composition for the solar set of element abundances is dominated by $\mathrm{TiO}_{2}[\mathrm{~s}]$ seed formation in the upper atmosphere. Other dust species start to condense on the seed decreasing its volume fraction steadily. A large fraction of the cloud layer is dominated by $\mathrm{Mg} / \mathrm{Si} / \mathrm{O}$-containing materials and below that (i.e. at higher temperatures) $\mathrm{Al}$ - and $\mathrm{Fe}$-containing species occupy a larger volume fraction by forming predominantly $\mathrm{Al}_{2} \mathrm{O}_{3}[\mathrm{~s}]$ and $\mathrm{Fe}[\mathrm{s}]$ until the particles become thermally unstable and evaporate completely. More details can be found in earlier pub- 


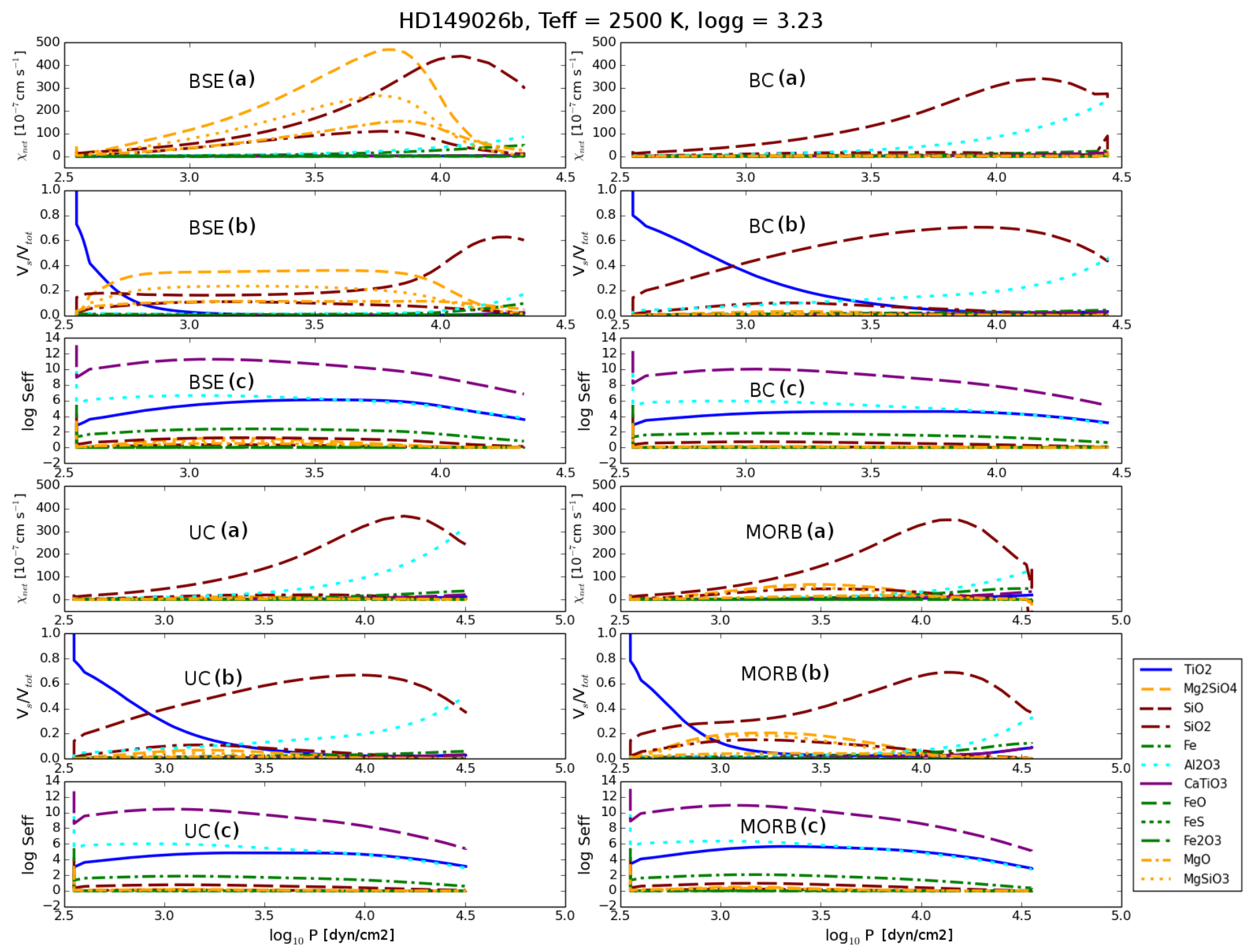

Figure 9. Same like Fig. 7 but for HD149026b $\left(\mathrm{T}_{\mathrm{eff}}=1757 \mathrm{~K}, \operatorname{logg}=3.23\right)$.

lications (e.g. Helling et al. 2008b; Helling \& Casewell 2014; Lee et al. 2015b; Helling et al. 2016b).

Figure 7 provides details about the variation of material composition of the cloud particles for non-solar sets of element abundances. We see an atmosphere with BSE composition to be consisting of Mg-binding materials ( 30\%), Si-binding materials ( $40 \%$ ) and finally $\mathrm{Al}_{2} \mathrm{O}_{3}[\mathrm{~s}]$ dominates as the primary dust species until a pressure of $10^{3} \mathrm{dyn} / \mathrm{cm}^{2}$ before the particles evaporate. The atmospheres with a BC, UC and MORB type of compositions have significant differences as compared to BSE, with the cloud particles being primarily composed of $\mathrm{SiO}[\mathrm{s}]$ and $\mathrm{SiO}_{2}[\mathrm{~s}]$ which in a combined manner constitute of more than $80 \%$ of the cloud volume fractions in each case. Hence, objects with a MORB-type (or $\mathrm{UC} / \mathrm{BC}$ ) set of initial element abundances can be expected to have mineral clouds predominantly made up of Si-O cloud particles with smaller impurities from high-temperature condensates.

\subsection{Clouds that form in the atmosphere of the metal-rich giant gas planet HD149 $026 \mathrm{~b}$}

HD149026 b can be classified as a mini-giant planet with a mass of $114 \mathrm{M}_{\oplus}$. Fortney et al. (2006b) investigate the atmospheric and cloud properties for the planet with varying metallicities such as $[\mathrm{M} / \mathrm{H}]$ of $1 \mathrm{x}, 3 \mathrm{x}, 10 \mathrm{x}$ with $\mathrm{TiO}$ and VO enrichment for $3 \mathrm{x}$ and $10 \mathrm{x}$. We perform our cloud modelling on a similar $\left(\mathrm{T}_{\text {gas }}, \mathrm{p}_{\text {gas }}\right)$ profile for $1 \mathrm{x}$ Solar abundance as shown in the Fig. 1 while varying the silicate abundances according to our four different Earth silicate compositions of BSE, BC, MORB and UC. Our models suggest a high value of vertical mixing with $\mathrm{K}_{z z}=10^{13} \mathrm{~cm}^{2} \mathrm{~s}^{-1}$ or higher is needed for the atmosphere to be able to form dust clouds.

Figures 8 and 9 show the dust cloud properties for HD149 026b as result of our kinetic cloud formation model. Also here, the cloud formation is triggered by the occurrence of $\mathrm{TiO}_{2}$ nucleation. Similar to previous findings or the giant gas planet atmosphere model, the nucleation rates $\left(J^{*}\right.$, panel 2 , left) differs by 1-2 orders of magnitude for the different sets of non-solar element abundances. The MORB composition produces the highest nucle- 


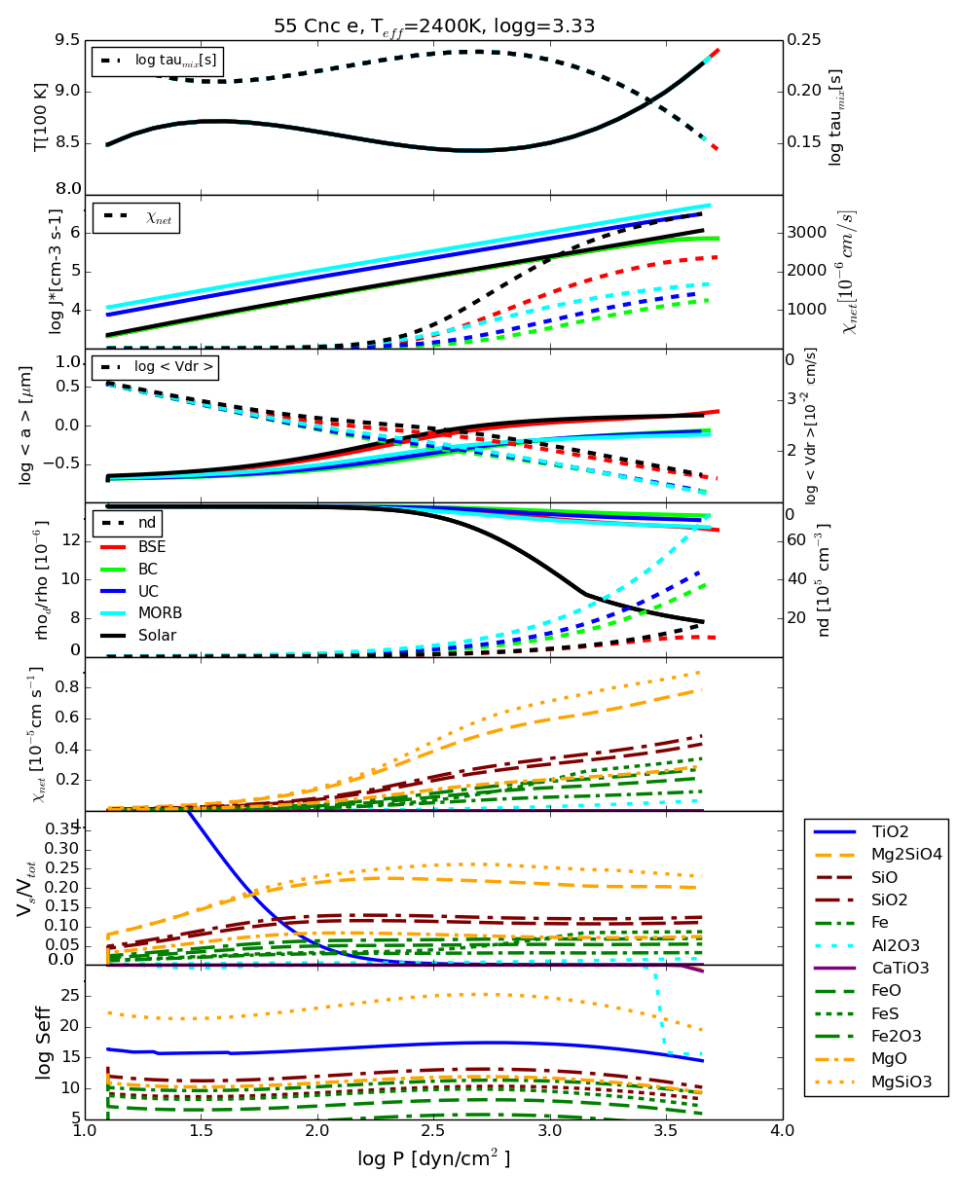

Figure 10. Cloud structure and cloud details for for $55 \mathrm{Cnc}$ e $\left(\mathrm{T}_{\mathrm{eff}}=2400 \mathrm{~K}\right.$, $\log g=3.33$ ) for five sets of initial element abundances (panel 2, 3, 4). The figure has the same structure like Fig. 6.

ation due to its higher Ti abundance (see Table 1). The particle growth velocities are the highest for a solar composition and the particles reach the largest sizes $(\sim 3 \mu \mathrm{m})$ followed by a BSE composition. The drift velocities $\left(\left\langle v_{d r}\right\rangle\right)$ are found to be in the same sequence of solar followed by BSE, MORB, BC and UC respectively. The particles formed from a solar composition gas are thermally stable in a larger range of pressure before they evaporate completely. The dust number densities $\left(\mathrm{n}_{\mathrm{d}}\right)$ are the highest for a MORB composition again as consequence of the highest $J_{*}$.

A solar and BSE composition in HD149026b follows a similar trend of dust composition wherein, just after the nucleation, Mg-binding silicates dominate the dust volume fraction with 40\% of the dust having Mg-binding dust species and is followed by $\mathrm{Si}$ ( $\sim 40 \%)$ and $\mathrm{Fe}-(\sim 30 \%)$ binding species which dominate the cloud base. For BC, UC and MORB compositions, our models suggest that the cloud particles majorly are made of Si-binding species ( $60 \%$ ) and the cloud base having an increased amount of $\mathrm{Al}_{2} \mathrm{O}_{3}$ [s] which increases up to $\sim 40 \%$ at the cloud base. Table 3 shows the average volume fractions for each of the dust forming species on the three of the possible atmospheres. Fortney et al. (2006b) consider atmospheric inversions due to the presence of $\mathrm{TiO}$ in their models but our dust cloud models suggest an upper atmosphere in which TiO is heavily depleted due to the seed formations. This is not a new finding and has been demonstrated for many cases (e.g. Helling et al. 2008b; Witte et al. 2009; Lee et al. 2015b). Such an element depletion would result in a loss of temperature inversion or suppression of the inversion zone to lower parts of the atmosphere where the $\mathrm{TiO}$ density is higher.

The cloud structure for HD149026b is comparable to the results for the hot giant gas planet (Sect. 4.1), but the cloud reaches considerably deeper into the atmosphere.

\subsection{Clouds in the atmosphere of 55 Cnc e}

Figures 10 and 11 show the cloud properties for $55 \mathrm{Cnc}$ e. Cloud formation only occurs in a thin atmospheric region near the top of the adopted $\left(\mathrm{T}_{\mathrm{gas}}, \mathrm{p}_{\mathrm{gas}}\right)$ profile where the local gas temperature and densities allow seed formation and efficient subsequent particle growth (compare pressure interval in Fig. 10 and Fig. 1). The extension of the cloud layer in the atmosphere of $55 \mathrm{Cnc} \mathrm{e}$ is therefore considerably smaller compared to the other cases studied here.

$\mathrm{TiO}_{2}$-nucleation occurs throughout the whole atmosphere domain available for $55 \mathrm{Cnc}$ e. Seed formation $\left(\mathrm{J}_{*}\right.$, panel 2, left) differs by about one order of magnitude for the different sets of element compositions, which effects the number of cloud particles formed accordingly. The MORB composition attains the highest nucleation rates which can be attributed to its higher abundance of Ti molecules leading to efficient nucleation. The sets of solar and $\mathrm{BC}$ element abundances results in the least efficient nucleation rate, hence, less cloud particles, $\mathrm{n}_{\mathrm{d}}$, are formed. The growth velocities (panel 2) are the highest for a solar composition followed by $\mathrm{BSE}, \mathrm{BC}, \mathrm{UC}$ and MORB. The particles encounter denser regions as they settle gravitationally and thereby also increase their size in the process due to availability of more reaction material. In the case of $55 \mathrm{Cnc}$ e, we observe a smooth change with height of the cloud material composition which is due to the fact that the cloud layer is thin as compared to the cloud layer in the gas giant. This, however, maybe a bias due to the domain of the prescribed atmosphere model available. The Solar and BSE compositions have Mg-binding silicates $(\sim 25 \%)$ for the major part of the dust cloud and it further increases to $\sim 30 \%$ of the dust before evaporation. Si-containing growth species $\mathrm{SiO}$ and $\mathrm{SiO}_{2}$ constitute $\sim 15 \%$ of the cloud particles. $\mathrm{Fe}, \mathrm{Al}$ and $\mathrm{Ca}$ species are present in minority $(<5 \%)$ in $\mathrm{BSE}$ atmosphere. $\mathrm{SiO}$ and $\mathrm{SiO}_{2}$ form the majority of the dust particles for $\mathrm{BC}, \mathrm{UC}$ and MORB atmospheres which is due to lower $\mathrm{Mg}$ content available to condense on the dust particles.

Our results for $55 \mathrm{Cnc}$ e demonstrate how sensible cloud formation reacts on the initial set of element abundances: solar abundances disfavour an efficient seed formation (based on $\mathrm{TiO}_{2}$ ) but allow for the most efficient surface growth processes among the sets of element abundances studied here. In contrast, also BC disfavours efficient nucleation but it also slows down surface growth being the least efficient in the sets studied. Consequently, the solar case produces clouds with few but big particles which rapidly rain out, and the cloud particles forming based on other sets remain suspended in the atmosphere for longer due to their smaller sizes.

\subsection{The possibility of clouds in the atmosphere of CoRoT-7b}

CoRoT-7b is an interesting candidate to analyze the possibility of cloud formation due to its extremely high local gas temperatures $(>2500 \mathrm{~K})$ and low local gas pressures $\left(-2<\log \left(p_{\text {gas }}\right)<\right.$ $\left.2\left[\mathrm{dyn} / \mathrm{cm}^{2}\right]\right)$ making it a challenging candidate for cloud formation based on the presently available $\left(\mathrm{T}_{\text {gas }}, \mathrm{p}_{\mathrm{gas}}\right)$-structures. Figure 1 demonstrates the large differences between those atmospheres where we have shown that clouds form and the atmosphere structures proposed for HRSE objects like CoRoT-7b. We investigated 

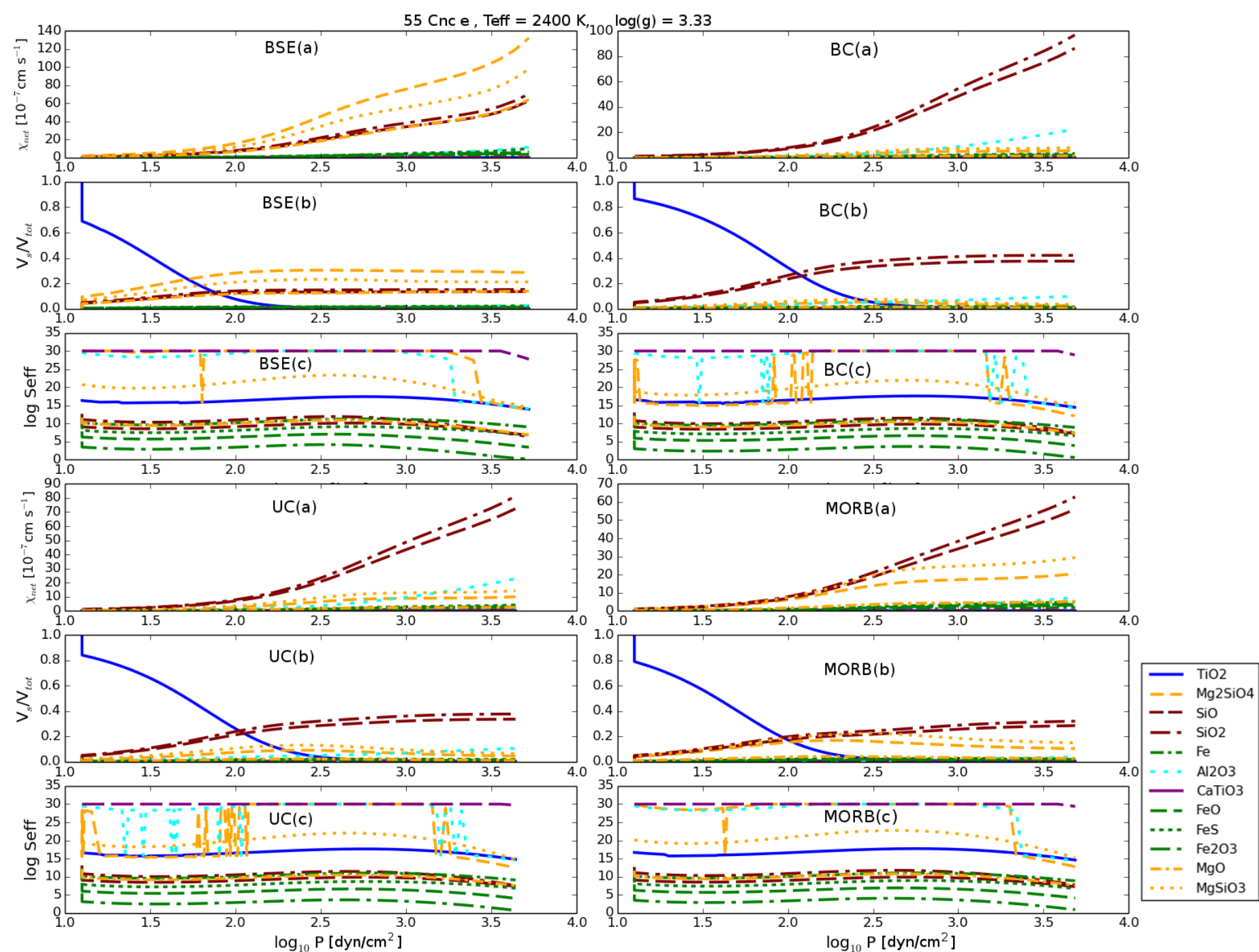

Figure 11. Same like Fig. 7 but for $55 \mathrm{Cnc}$ e $\left(\mathrm{T}_{\text {eff }}=2400 \mathrm{~K}, \operatorname{logg}=3.33\right)$.

if cloud formation is possible on CoRoT-7b's day-side by using the sample atmosphere profiles for a rocky planet with four different $\mathrm{T}_{\text {eq }}$ (Fig. 1). Our kinetic cloud model does not suggest any cloud formation on the day-side of CoRoT-7b for the atmosphere structures provided. Our finding, however, does not preclude cloud formation on the night side.

Schaefer \& Fegley (2009) predict clouds of $\mathrm{Na}$ and $\mathrm{K}$ in the planet's atmosphere based on their phase-equilibrium approach. However, Gao \& Benneke (2016) show that it can not be taken for granted that such species actually nucleate, and argument that has been put forward in Helling \& Fomins (2013). Phase-equilibrium approaches focus on possible end-results of condensation processes but do not offer insight into the process of formation (Helling et al. 2008a). The $\left(\mathrm{T}_{\mathrm{gas}}, \mathrm{p}_{\mathrm{gas}}\right)$-structures utilized here for CoRoT-7b appear hot enough that a strong ionisation of the atmospheric gas should be expected according to our gas-phase composition study in Sect. 3. Given the high irradiation onto an existing atmosphere, strong winds will develop and transport ionised gas into cooler atmospheric regions where clouds will form similar to $55 \mathrm{Cnc} \mathrm{e}$. The night-side/day-side transition region will most likely be electrostat- ically active and electric currents are likely to affect the weather on CoRot 7b as suggested in e.g. the review by Helling et al. (2016b).

\subsection{Summary}

Table 3 provides an overview of global changes of the cloud structure depending on the sets of initial element abundances. We chose to visualise this in terms of volume fractions $\left(\mathrm{V}_{\mathrm{s}} / \mathrm{V}_{\text {tot }}[\%]\right)$ at three typical heights inside the cloud, the maximum nucleation rate, and the average mean grain size throughout the $1 \mathrm{D}$ cloud layer. These values can serve as guidance for retrieval methods.

The highlighted values for $\mathrm{V}_{\mathrm{s}} / \mathrm{V}_{\text {tot }}$ visualise that several materials dominate the cloud particle composition but the type of material chances with height in all cases studied. Table 3 demonstrates further that a number of materials do contribute with less than $10 \%$ to the overall cloud particle volume $\left(\mathrm{CaTiO}_{3}[\mathrm{~s}], \mathrm{Fe}_{2} \mathrm{O}_{3}[\mathrm{~s}], \mathrm{FeS}[\mathrm{s}]\right.$, $\mathrm{FeO}[\mathrm{s}])$.

The nucleation species $\mathrm{TiO}_{2}[\mathrm{~s}]$ dominates the cloud particle composition at the cloud top while $\mathrm{Mg}-\mathrm{Si}-\mathrm{O}$ materials provide the material bulk independent on the set of initial element abundances. 
We use the maximum nucleation rate and the average mean grain size as measured to compare our results for the individual objects (hot gas giant, HD 149026b, $55 \mathrm{Cnc}$ e). This clearly shows that the effect of the different initial element abundances are negligible compared to differences in local thermodynamic conditions. The anti-correlation between nucleation rate (hence, number of cloud particles formed) and mean grain size outlined earlier prevails also globally.

CoRot7b has no clouds forming on its day side and a strong day-side/night-side contrast should therefore be expected which is determined by the planet's cloud formation.

\section{CONCLUSIONS}

Element abundances are important input properties for atmosphere (and evolutionary) modelling which are, however, rarely known. Determining element abundances for extrasolar planets would be a valuable exercise as this might allow to back-trace their place inside a planet-forming disk. However, chemical processing inside the protoplanetary disk and in the planet's atmosphere need to be known well enough for this exercise.

This work is the first attempt to model cloud formations in planetary atmospheres with Earth-silicate like element compositions. Our equilibrium chemistry gas-phase composition reflects the adopted silicate element compositions from the rocky and the solar element abundances. Apart from the volatile rich atmosphere consisting primarily of $\mathrm{H}_{2}, \mathrm{H}$ and $\mathrm{CO}$ there is an increase in content of gases such as $\mathrm{Mg}, \mathrm{Fe}, \mathrm{SiS}, \mathrm{Al}, \mathrm{Ca}, \mathrm{Si}, \mathrm{Ti}$ and $\mathrm{K}$.

Our results suggest that clouds form in the atmospheres of 55 Cnc e, HD149 026b, and also in the hot gas giant as we demonstrated in previous works. Cloud formation in atmospheres of evaporating magma planets (referring to planets with a molten surface) like $55 \mathrm{Cnc}$ e requires a vertical recirculation of condensible material of $>10^{11} \mathrm{~cm}^{2} \mathrm{~s}^{-1}$. Vertical replenishment mechanisms have not been explored in detail in this work but a large day-night temperature gradient may favour a circulation mechanism and hence increase the likeliness of cloud formation at or near the sub-stellar point. Such possibilities have not been ruled out by Demory et al. (2016b) wherein the large observed temperature gradient between the day and night side may indeed favour cloud formation. They also find the hotspot to be shifted by $41 \pm 12$ degrees towards the east of the sub-solar point which further augments the possibility of a strong circulation regime. Our cloud model indicates the possibility of a highly opaque cloud layer on 55 Cancri-e with particles primarily consisting of $\mathrm{Mg}$ silicates ( 25\%), followed by Si-oxides and a minor percentage of $\mathrm{Fe}-, \mathrm{Al}-$ and $\mathrm{Ca}-$ oxides. Recent spectroscopic observations of 55 Cancri-e by Tsiaras et al. (2015) suggest an atmosphere which has $\mathrm{C} / \mathrm{O} \sim 1$ having a high concentration of HCN.

Our mineral cloud formation models show a large variation in particle sizes and compositions for different planetary scenarios and different sets of element abundances as tabulated in Table 3. The particle sizes are found to be the largest in the case of giant gas planet which is indicative of the denser atmosphere resulting in more condensing material availability. The particle sizes follow the order of Gas Giant > HD149 026b > 55 Cnc e, with the largest particles found at the cloud base in each of the cases below which the particles evaporate. The biggest particle sizes are $54 \mu \mathrm{m}$ for the solar case, $3.6 \mu \mathrm{m}$ for the BSE case, and $3 \mu \mathrm{m}$ for the BC Giant gas planet case. These values change for $55 \mathrm{Cnc}$ e to $0.5 \mu \mathrm{m}$, $0.47 \mu \mathrm{m}$ and $0.32 \mu \mathrm{m}$ for the solar, BSE and BC cases, respectively. For HD149 026b, we find maximum mean cloud particle radii of $0.6 \mu \mathrm{m}, 0.4 \mu \mathrm{m}$, and $0.28 \mu \mathrm{m}$ solar, BSE and BC cases, respectively. The cloud particle number densities $\left(\mathrm{n}_{\mathrm{d}}\left[\mathrm{cm}^{-3}\right)\right.$ are 10 times higher for the atmosphere of $55 \mathrm{Cnc}$ e as compared to HD149 026b which will contribute to a higher cloud opacity on $55 \mathrm{Cnc}$ e.

We observe a similarity in the cloud property trend due to the changes in element abundances on each of the planets cases where cloud formation happens. The particle sizes are found to be maximum with a solar and BSE composition for each of the planets. This is due to higher $\mathrm{Mg}$ and $\mathrm{O}$ content in the atmosphere with these abundances which results in faster material growth of species such as $\mathrm{MgSiO}_{3}[\mathrm{~s}]$ and $\mathrm{Mg}_{2} \mathrm{SiO}_{4}[\mathrm{~s}]$. The dust number density $\left(\mathrm{n}_{\mathrm{d}}\right)$ is found to be the highest for a MORB atmosphere which can be explained due to higher Ti content which results in more seed species $\left(\mathrm{TiO}_{2}\right)$ formation during the nucleation stage. The particle composition also follows a similar trend due to changes in element abundances. Mineral cloud particles in BC, UC and MORB atmospheres predominantly constitute of $\mathrm{SiO}[\mathrm{s}]$ and $\mathrm{SiO}_{2}[\mathrm{~s}]$ species and their percentages vary proportionally with the changes in element abundances. Similarly a BSE and solar atmosphere would have dust particles predominantly of $\mathrm{Mg}_{2} \mathrm{SiO}_{4}[\mathrm{~s}], \mathrm{MgSiO}_{3}$ [s] and $\mathrm{MgO}[\mathrm{s}]$. Only the atmosphere of gas giant has pressures and temperatures suitable for cloud base to form particles of $\mathrm{Al}_{2} \mathrm{O}_{3}[\mathrm{~s}]$ and $\mathrm{Fe}[\mathrm{s}]$ species. Lee et al. (2015b) in their analysis for 3D cloud formations on HD189733b find similar high volume fractions of $\mathrm{Fe}-$ and Al-binding in their cloud particles and suggest a locally lower cloud opacity due to $\mathrm{Al}_{2} \mathrm{O}_{3}[\mathrm{~s}]$ and high opacity due to $\mathrm{Fe}[\mathrm{s}]$ which could alter the radiation propagation.

To summarize the results obtained in this work:

- Our models suggest possibility of mineral cloud formations on 55 Cnc e and HD149 026b. The atmosphere of CoRoT-7b or HRSE is found to be too warm on the day-side for gas condensation to occur based on the atmosphere profile applied.

- Our results indicate that changes in element abundance compositions result in significant changes in the compositions of (mineral) cloud particles. Solar and BSE atmospheres consists primarily of $\mathrm{Mg}$ materials whereas $\mathrm{BC}$, UC and MORB atmospheres consist of $\mathrm{Si}$ - and $\mathrm{Fe}$-binding materials

- The cloud particle properties for different compositions follow a trend of variation independent of local gas-phase pressures and temperatures. As an example, an atmosphere with high abundance of a seed forming species, such as Ti that is found in MORB composition, will have a larger cloud number density as compared to $\mathrm{BSE}, \mathrm{BC}$ and UC compositions due to higher number of seed particles formed.

- The atmospheres of $55 \mathrm{Cnc}$ e and HD149 026b requires strong element replenishment to be able to sustain cloud formation in the atmosphere. An atmosphere with no vertical replenishment results in the heavy elements raining out, rendering the atmosphere depleted of heavy elements.

Magma (or volcano) planets will form thick opaque clouds that will effect the evolution of these rocky planets, and with that maybe also the emergence of plate tectonics. With the bulk element abundances for extrasolar planets being very difficult to determine from present formation and evolutionary models, Table 3 provides global mean values for cloud properties for various rocky 
Table 3. A comparison of characteristic cloud properties for a gas giant, $55 \mathrm{Cnc}$ e and HD 149026b: the volume fractions ( $\mathrm{V}_{\mathrm{s}} / \mathrm{V}_{\text {tot }}[\%]$ ) for individual materials $s$, the maximum nucleation rates $\left(\log _{10} \mathrm{~J}_{*, \max }\left[\mathrm{cm}^{-3} \mathrm{~s}^{-1}\right]\right)$ and cloud-averaged mean cloud particle radius $\left(\langle a\rangle_{\text {avg }}[\mu \mathrm{m}]\right)$ for three types of compositions. The cloud properties are compared for the cloud top, the middle (approximate half-length of the cloud) and for the cloud base (where the cloud particles have evaporated). All $\mathrm{V}_{\mathrm{s}} / \mathrm{V}_{\text {tot }}>10 \%$ are shown in boldface.

\begin{tabular}{|c|c|c|c|c|c|c|c|c|c|c|}
\hline \multirow{2}{*}{$\begin{array}{c}\text { Volume fraction } V_{\mathrm{s}} / V_{\text {tot }}[\%] \\
\mathrm{p}_{\mathrm{gas}}[\mathrm{bar}]\end{array}$} & \multirow[t]{2}{*}{$\epsilon_{\mathrm{i}}^{0}$} & \multicolumn{3}{|c|}{ Gas Giant } & \multicolumn{3}{|c|}{55 Cnc e } & \multicolumn{3}{|c|}{ HD149026b } \\
\hline & & $\begin{array}{l}\text { Cloud Top } \\
10^{-5.5}\end{array}$ & $\begin{array}{l}\text { Middle } \\
10^{1.3}\end{array}$ & $\begin{array}{c}\text { Base } \\
10^{3}\end{array}$ & $\begin{array}{l}\text { Cloud Top } \\
10^{6.04}\end{array}$ & $\begin{array}{c}\text { Middle } \\
10^{6.4}\end{array}$ & $\begin{array}{l}\text { Base } \\
10^{6.7}\end{array}$ & $\begin{array}{l}\text { Cloud Top } \\
10^{2.5}\end{array}$ & $\begin{array}{c}\text { Middle } \\
10^{3}\end{array}$ & $\begin{array}{l}\text { Base } \\
10^{5.2}\end{array}$ \\
\hline \multirow{3}{*}{$\mathrm{TiO}_{2}[\mathrm{~s}]$} & Solar & 100.0 & 0.04 & 1.66 & 0.4 & 0.1 & 5.05 & 99.6 & 2.2 & 1 \\
\hline & BSE & 100.0 & 0.1 & 4.47 & 1.67 & 0.13 & 6.4 & 99.9 & 4.61 & 2.2 \\
\hline & BulkCrust & 100.0 & 0.3 & 5.8 & 29 & 0.9 & 9.9 & 99.9 & 45.2 & 3.02 \\
\hline \multirow{3}{*}{$\mathrm{Al}_{2} \mathrm{O}_{3}[\mathrm{~s}]$} & Solar & 0 & 2.79 & 97.4 & 0.7 & 1.07 & 3.9 & 0.002 & 0.8 & 13.4 \\
\hline & BSE & 0 & 6 & 94.2 & 0.9 & 1.35 & 6.13 & $<0.001$ & 1.2 & 17 \\
\hline & BulkCrust & 0 & 17.7 & 93.1 & 3.6 & 6.3 & 16 & $<0.01$ & 6.7 & 45 \\
\hline \multirow{3}{*}{$\mathrm{CaTiO}_{3}[\mathrm{~s}]$} & Solar & 0 & 0.15 & 0.47 & 0.01 & 0.01 & 0.35 & $<0.001$ & 0.05 & 1.1 \\
\hline & BSE & 0 & 0.16 & 1 & 0.03 & 0.05 & 0.35 & 0 & 0.07 & 1.0 \\
\hline & BulkCrust & 0 & 0.7 & 0.7 & 0.13 & 0.16 & 0.96 & 0 & 0.4 & 2.6 \\
\hline \multirow{3}{*}{$\mathrm{Fe}_{2} \mathrm{O}_{3}[\mathrm{~s}]$} & Solar & $<0.001$ & $<0.001$ & $<0.001$ & 5.15 & 6 & $<0.001$ & $<0.001$ & $<0.001$ & 0 \\
\hline & BSE & 0 & 0 & 0 & 0.3 & 0.33 & 0 & 0 & 0 & 0 \\
\hline & BulkCrust & 0 & 0 & 0 & 0.08 & 0.14 & 0 & 0 & 0 & 0 \\
\hline \multirow{3}{*}{$\mathrm{FeS}[\mathrm{s}]$} & Solar & 0 & 0.08 & $<0.01$ & 4.8 & 8.02 & 0.2 & 0.01 & 1.4 & 0.1 \\
\hline & BSE & 0 & 0.05 & 4.2 & 1.7 & 2 & 0.1 & $<0.001$ & 0.15 & 0.04 \\
\hline & BulkCrust & 0 & $<0.01$ & 0 & 1.36 & 1.86 & 0.02 & 0 & 0.04 & $<0.01$ \\
\hline \multirow{3}{*}{$\mathrm{FeO}[\mathrm{s}]$} & Solar & 0 & $<0.01$ & $<0.01$ & 5.6 & 5.6 & 0.2 & 0.01 & 0.5 & 0.05 \\
\hline & BSE & 0 & 0 & $<0.001$ & 1.11 & 1.25 & 0.02 & $<0.001$ & 0.01 & $<0.01$ \\
\hline & BulkCrust & 0 & 0 & 0 & 0.85 & 1.16 & $<0.01$ & 0 & $<0.01$ & $<0.01$ \\
\hline \multirow{3}{*}{$\mathrm{Fe}[\mathrm{s}]$} & Solar & 0 & 14.6 & 0.34 & 3.3 & 3.3 & 18.1 & 0.01 & 4 & 41.7 \\
\hline & BSE & 0 & 4.11 & 0.14 & 0.6 & 0.7 & 4 & 0 & 0.8 & 9.5 \\
\hline & BulkCrust & 0 & 2.2 & 0.05 & 0.5 & 0.7 & 1.9 & 0 & 0.8 & 4.2 \\
\hline \multirow{3}{*}{$\mathrm{SiO}[\mathrm{s}]$} & Solar & 0 & 9.7 & 0.08 & 12.4 & 11.7 & 46.4 & 0.05 & 15 & 28 \\
\hline & BSE & 0 & 45.9 & 0.16 & 13.4 & 13.4 & 66.4 & $<0.001$ & 16.2 & 60.1 \\
\hline & BulkCrust & 0 & 72.3 & 0.16 & 25 & 36.6 & 69.8 & $<0.01$ & 35.6 & 44.5 \\
\hline \multirow{3}{*}{$\mathrm{SiO}_{2}$} & Solar & 0 & 14.8 & $<0.001$ & 14 & 13.2 & 3.5 & 0.05 & 15.7 & 2.8 \\
\hline & BSE & 0 & 1.01 & $<0.001$ & 15.1 & 15 & 2.32 & $<0.01$ & 10.0 & 2.0 \\
\hline & BulkCrust & 0 & 3.14 & $<0.01$ & 28.1 & 41.1 & 0.8 & $<0.01$ & 7.4 & 0.5 \\
\hline \multirow{3}{*}{$\mathrm{MgO}[\mathrm{s}]$} & Solar & 0 & 5.1 & $<0.001$ & 9.1 & 8.35 & 10.5 & 0.03 & 11 & 6.1 \\
\hline & BSE & 0 & 15.8 & $<0.001$ & 12.3 & 12.5 & 9 & $<0.01$ & 10.5 & 5.0 \\
\hline & BulkCrust & 0 & 0.6 & $<0.01$ & 1.07 & 1.05 & 0.18 & 0 & 0.5 & 0.07 \\
\hline \multirow{3}{*}{$\mathrm{MgSiO}_{3}[\mathrm{~s}]$} & Solar & 0 & 22.4 & $<0.001$ & 23.1 & 23.1 & 3.2 & 0.07 & 24.2 & 2 \\
\hline & BSE & 0 & 6.05 & 0 & 22.4 & 22.7 & 1.9 & $<0.01$ & 22.1 & 1.3 \\
\hline & BulkCrust & 0 & 0.6 & 0 & 6.0 & 5.9 & $<0.01$ & $<0.01$ & 1.0 & $<0.01$ \\
\hline \multirow{3}{*}{$\mathrm{Mg}_{2} \mathrm{SiO}_{4}[\mathrm{~s}]$} & Solar & 0 & 30.2 & $<0.001$ & 21.3 & 19.4 & 8.3 & 0.07 & 25.4 & 3.6 \\
\hline & BSE & 0 & 20.7 & 0 & 30.1 & 30.3 & 3.3 & $<0.01$ & 34.1 & 1.6 \\
\hline & BulkCrust & 0 & 2.2 & 0 & 4.17 & 4.1 & 0.13 & $<0.01$ & 2.06 & 0.05 \\
\hline \multirow{3}{*}{$\begin{array}{l}\text { Maximum nucleation rate } \\
\log _{10} \mathrm{~J}_{*, \max }\left[\mathrm{cm}^{-3} \mathrm{~s}^{-1}\right]\end{array}$} & Solar & & -8.1 & & & 12.13 & & & 5.98 & \\
\hline & BSE & & -9.75 & & & 11.86 & & & 4.42 & \\
\hline & BulkCrust & & -8.91 & & & 12.35 & & & 4.74 & \\
\hline \multirow{3}{*}{$\begin{array}{l}\text { Cloud-averaged mean cloud particle radius } \\
\qquad\langle a\rangle_{\mathrm{avg}}[\mu \mathrm{m}]\end{array}$} & Solar & & 2.83 & & & 0.50 & & & 0.58 & \\
\hline & BSE & & 3.58 & & & 0.47 & & & 0.43 & \\
\hline & BulkCrust & & 2.98 & & & 0.32 & & & 0.29 & \\
\hline
\end{tabular}

abundances as a first guidance for retrieval methods and for planet evolution model.

\section{ACKNOWLEDGEMENTS}

We thank the referee for providing helpful feedback which led to further improvements of this paper. GM acknowledges an ERASMUS studentship from the TU Delft. ChH highlight financial sup- port of the European Community under the FP7 by an ERC starting grant number 257431. YM greatly appreciates the CNES travel funding and post-doctoral fellowship program. We are grateful to J. Fortney for finding us the HD149026b atmosphere structures. We would like to thank Paul Rimmer for useful discussions that helped to improve the manuscript. 


\section{REFERENCES}

Agúndez M., Parmentier V., Venot O., Hersant F., Selsis F., 2014, Astronomy \& Astrophysics, 564, A73

Bilger C., Rimmer P., Helling C., 2013, MNRAS, 435, 1888

Cridland A. J., Pudritz R. E., Alessi M., 2016, MNRAS, 461, 3274

Demory B.-O., et al., 2011, Astronomy \& Astrophysics, 533, A114

Demory B.-O., Gillon M., Seager S., Benneke B., Deming D., Jackson B., 2012, The Astrophysical Journal Letters, 751, L28

Demory B.-O., Gillon M., Madhusudhan N., Queloz D., 2016a, Monthly Notices of the Royal Astronomical Society, 455, 2018

Demory B.-O., et al., 2016b, Nature, 532, 207

Dressing C. D., et al., 2015, ApJ, 800, 135

Eistrup C., Walsh C., van Dishoeck E. F., 2016a, A\&A, 595, A83

Eistrup C., Walsh C., van Dishoeck E. F., 2016b, A\&A, 595, A83

Fegley B., Jacobson N. S., Williams K., Plane J., Schaefer L., Lodders K., 2016, The Astrophysical Journal, 824, 103

Fortney J. J., Saumon D., Marley M. S., Lodders K., Freedman R. S., 2006a, ApJ, 642, 495

Fortney J., Saumon D., Marley M., Lodders K., Freedman R., 2006b, The Astrophysical Journal, 642, 495

Fortney J. J., Lodders K., Marley M. S., Freedman R. S., 2008, ApJ, 678, 1419

Gao P., Benneke B., 2016, in AAS/Division for Planetary Sciences Meeting Abstracts. p. 302.02

Gillon M., et al., 2012, Astronomy \& Astrophysics, 539, A28

Grevesse N., Asplund M., Sauval A., 2007, Space Science Reviews, 130, 105

Guillot T., Burrows A., Hubbard W., Lunine J., Saumon D., 1996, The Astrophysical Journal Letters, 459, L35

Helling C., Casewell S., 2014, The Astronomy and Astrophysics Review, 22,1

Helling C., Fomins A., 2013, Philosophical Transactions of the Royal Society of London Series A, 371, 20110581

Helling C., Woitke P., 2006, A\&A, 455, 325

Helling C., et al., 2008a, MNRAS, 391, 1854

Helling C., Woitke P., Thi W.-F., 2008b, Astronomy \& Astrophysics, 485, 547

Helling C., Woitke P., Rimmer P. B., Kamp I., Thi W.-F., Meijerink R., 2014, Life, 4

Helling C., et al., 2016a, Surveys in Geophysics, 37, 705

Helling C., et al., 2016b, MNRAS, 460, 855

Ito Y., Ikoma M., Kawahara H., Nagahara H., Kawashima Y., Nakamoto T., 2015, The Astrophysical Journal Letters, 801, 144

Jeong K. S., Winters J. M., Sedlmayr E., 1999, in Le Bertre T., Lebre A., Waelkens C., eds, IAU Symposium Vol. 191, Asymptotic Giant Branch Stars. p. 233

Jeong K. S., Chang C., Sedlmayr E., Sülzle D., 2000, Journal of Physics B Atomic Molecular Physics, 33, 3417

Khodachenko M. L., Shaikhislamov I. F., Lammer H., Prokopov P. A., 2015, ApJ, 813, 50

Kirkby J., et al., 2011, Nature, 476, 429

Knutson H. A., Benneke B., Deming D., Homeier D., 2014, Nature, 505, 66

Lee G., Helling C., Giles H., Bromley S. T., 2015a, A\&A, 575, A11

Lee G., Helling C., Dobbs-Dixon I., Juncher D., 2015b, Astronomy \& Astrophysics, 580, A12

Lopez E. D., Rice K., 2016, preprint, (arXiv: 1610.09390)

Madhusudhan N., 2012, The Astrophysical Journal, 758, 36

McDonough W., s. Sun S., 1995, Chemical Geology, 120, 223

Miguel Y., Kaltenegger L., 2013, The Astrophysical Journal, 780, 166

Miguel Y., Kaltenegger L., Fegley B., Schaefer L., 2011, The Astrophysical Journal Letters, 742, L19

Mordasini C., van Boekel R., Mollière P., Henning T., Benneke B., 2016, ApJ, 832, 41

Moses J. I., et al., 2011, The Astrophysical Journal, 737, 15

Nelson B. E., Ford E. B., Wright J. T., Fischer D. A., von Braun K., Howard A. W., Payne M. J., Dindar S., 2014, Monthly Notices of the Royal Astronomical Society, 441, 442
Owen J. E., Mohanty S., 2016, MNRAS, 459, 4088

Palme H., Lodders K., Jones A., 2013, Treatise on Geochemistry: Second Edition, 2, 15

Parmentier V., Showman A. P., Lian Y., 2013, Astronomy \& Astrophysics, 558, A91

Rimmer P. B., Helling C., Bilger C., 2014, International Journal of Astrobiology, 13, 173

Rogers L. A., 2015, ApJ, 801, 41

Sato B., et al., 2005, The Astrophysical Journal, 633, 465

Schaefer L., Fegley B., 2004, icarus, 169, 216

Schaefer L., Fegley B., 2009, The Astrophysical Journal Letters, 703, L113

Schaefer L., Lodders K., Fegley B., 2012, The Astrophysical Journal, 755, 41

Sudarsky D., Burrows A., Hubeny I., Li A., 2005, ApJ, 627, 520

Taylor S. R., McLennan S., 2009, Planetary Crusts: Their Composition, Origin and Evolution

Trammell G. B., Arras P., Li Z.-Y., 2011, ApJ, 728, 152

Tsiaras A., et al., 2015, arXiv preprint arXiv:1511.08901

Winn J. N., et al., 2011, The Astrophysical Journal Letters, 737, L18

Witte S., Helling C., Hauschildt P., 2009, Astronomy \& Astrophysics, 506, 1367

Witte S., Helling C., Barman T., Heidrich N., Hauschildt P., 2011, Astron. Astrophys., 529, A44

Woitke P., Helling C., 2003, A\&A, 399, 297

Woitke P., Helling C., 2004, A\&A, 414, 335

\section{APPENDIX A: ELEMENT ABUNDANCES AND GROWTH SURFACE REACTIONS}

\section{A1 Terrestrial element abundances from rocks}

The composition of a rock is expressed in terms of weight oxides in the geoscience literature. This is expressed in weight percentage of a particular compound, for example the upper crust of the Earth is a mixture of $66.6 \% \mathrm{SiO}_{2}$ [s], $15.4 \% \mathrm{Al}_{2} \mathrm{O}_{3}[\mathrm{~s}], 3.59 \% \mathrm{CaO}[\mathrm{s}]$. In astronomical literature, element abundances are expressed relative to $10^{12} \mathrm{H}$ atoms such the the element abundance $\epsilon_{\mathrm{i}}$ for a certain species $i$ is $(\mathrm{i}=\mathrm{Si}, \mathrm{O}, \mathrm{Mg}, \ldots)$

$$
\log \left(\epsilon_{\mathrm{i}}\right)=\log \left(\frac{\mathrm{n}_{\mathrm{i}}}{\mathrm{n}_{\mathrm{H}}}\right)+12 .
$$

$n_{\mathrm{i}}\left[\mathrm{cm}^{-3}\right]$ is the number density of the element $i$, hence, $\mathrm{n}_{\mathrm{H}}=10^{12}$. The weight oxide $(\%)$ of each material $\left(\mathrm{SiO}_{2}[\mathrm{~s}], \mathrm{FeO}[\mathrm{s}], \mathrm{Al}_{2} \mathrm{O}_{3}[\mathrm{~s}]\right.$ etc.) is used to calculate the number of moles of that compound in a particular rock type as

$$
\mathrm{M}(\text { No.ofmoles })=\frac{\mathrm{m}(\% \text { of Compound })}{\mathrm{n}(\text { molar mass }[\mathrm{g} / \mathrm{mole}])} .
$$

The number of moles of a particular element, e.g. oxygen $(\mathrm{O})$ in $\mathrm{SiO}_{2}[\mathrm{~s}]$ is calculated as

$$
\mathrm{M}_{\mathrm{O}}=v_{\mathrm{O}} \cdot \mathrm{M}
$$

$v_{\mathrm{i}}$ is the stoichiometric coefficient of the chosen element in the compound. The total number of atoms of a particular element can then be derived from

$$
\mathrm{N}_{\mathrm{tot}, \mathrm{i}}=\mathrm{N}_{\mathrm{A}} \sum v_{\mathrm{s}} \mathrm{M}_{\mathrm{i}, \mathrm{s}} .
$$

$\mathrm{n}_{\text {tot, } \mathrm{i}}$ is the total number of moles of a particular element in the mixtures, $s$ is the compound $\left(\mathrm{SiO}_{2}[\mathrm{~s}], \mathrm{Al}_{2} \mathrm{O}_{3}\right.$ [s], $\mathrm{FeO}[\mathrm{s}]$ etc.) and $\mathrm{N}_{A}$ [atoms/mole] is Avogadro's number.

The rock composition in Tables A1, 1, do not contain information about potential hydrogen content that a rocky planet's atmosphere may have. Thus, we follow the method generally used to 


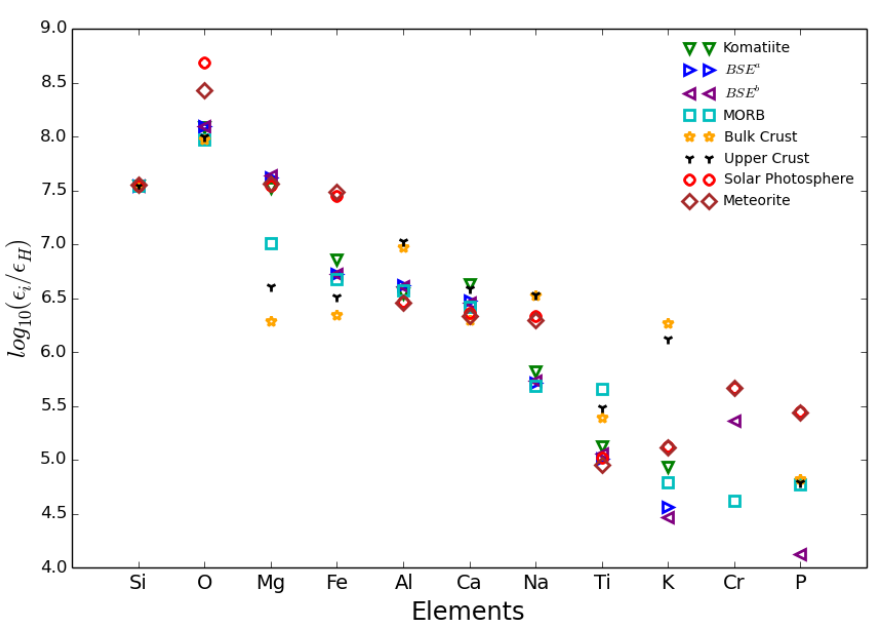

Figure A1. Element abundance $\left(\log _{10}\left(\epsilon_{\mathrm{H}}\right)=12\right.$ scale), for six Earth rock and magma composition. The komatiite and $\mathrm{BSE}^{a}$ compositions are used by Miguel et al. 2011 and $\mathrm{BSE}^{b}$, MORB, Bulk Crust and Upper Crust compositions are used by Ito et al. 2015 (see Tables A1, 1). The solar photospheric and meteorites abundances are shown for comparison (Grevesse et al. 2007).

calculate the element abundances from meteorites where the abundance ratios are calculated with respect to the $\mathrm{Si}$ atoms in a scale of $\log _{10} \epsilon_{\mathrm{Si}}=6$,

$$
\log \left(\epsilon_{\mathrm{Si}}\right)=\log \left(\frac{\mathrm{n}_{\mathrm{tot}, \mathrm{i}}}{n_{\mathrm{Si}}}\right)+6 .
$$

To be able to convert the Si-normalized abundances to a $\mathrm{H}$ normalized abundances scale, we follow the method outlined by Palme et al. (2013). The conversion factor between the two scales was calculated by dividing the H-normalized solar abundances by the Si-normalized meteorite abundances. The comparison was made for all elements with an error of the corresponding photospheric abundance of less than 0.1 dex, i.e., less than $25 \%$. The log of the average ratio of solar abundance per $10^{12} \mathrm{H}$ atoms/meteorite abundance per $10^{6}$ silicon atoms is $1.546 \pm 0.045$ (Palme et al. 2013). Thus,

$$
\log \left(\epsilon_{\mathrm{H}}\right)=\log \left(\epsilon_{\mathrm{Si}}\right)+1.546
$$

The resulting element abundances in the $\log \left(\epsilon_{H}\right)=12$ scale are listed in Table 1. In Fig. A1, we compare the element abundances derived for all the six types of rocks (bulk crust - yellow stars, upper crust - black, MORB - light-blue squares (metal-oxide-ridge basalt), BSE ${ }^{\mathrm{a}}$ - blue triangle (bulk silicate Earth), $\mathrm{BSE}^{\mathrm{b}}$ - purple triangle, komatite - green triangle). We include a comparison to the solar (red circles) and the meteorite (brown diamonds) element abundances.

\section{A2 Surface-growth reactions}

This paper has been typeset from a $\mathrm{T}_{\mathrm{E}} \mathrm{X} / \mathrm{L} \mathrm{AT} \mathrm{E} \mathrm{X}$ file prepared by the author. 
Table A1. Weight (\%) of oxides for various types of Earth and magma rocks.

\begin{tabular}{ccccccc}
\hline \hline Weight oxide (\%) & Komatite $^{a}$ & BSE $^{b}$ & BSE $^{c}$ & MORB $^{c}$ & Upper Crust $^{d}$ & Bulk Crust $^{d}$ \\
\hline $\mathrm{SiO}_{2}$ & 47.10 & 45.97 & 45.1 & 49.6 & 66.6 & 60.6 \\
$\mathrm{MgO}$ & 29.60 & 36.66 & 37.9 & 9.75 & 2.5 & 4. \\
$\mathrm{FeO}$ & - & 8.24 & 8.06 & 8.06 & 5.04 & 6.71 \\
$\mathrm{Al}_{2} \mathrm{O}_{3}$ & 4.04 & 4.77 & 4.46 & 16.8 & 15.4 & 15.9 \\
$\mathrm{CaO}$ & 5.44 & 3.78 & 3.55 & 12.5 & 3.59 & 6.40 \\
$\mathrm{Na} 2$ & 0.46 & 0.35 & 0.36 & 2.18 & 3.27 & 3.07 \\
$\mathrm{Cr}_{2} \mathrm{O}_{3}$ & - & - & 0.38 & 0.07 & 0.0003 & 0.0004 \\
$\mathrm{TiO}_{2}$ & 0.24 & 0.18 & 0.2 & 0.9 & 0.64 & 0.72 \\
$\mathrm{~K}_{2} \mathrm{O}$ & 0.09 & 0.04 & 0.03 & 0.07 & 2.8 & 1.81 \\
$\mathrm{P}_{2} \mathrm{O}_{5}$ & - & - & 0.02 & 0.1 & 0.13 & 0.15 \\
$\mathrm{Fe}_{2} \mathrm{O}_{3}$ & 12.8 & - & - & - & - & - \\
\hline
\end{tabular}

${ }^{a}$ Schaefer \& Fegley (2004), ${ }^{b}$ O’Neill \& Palme (1998), ${ }^{c}$ McDonough \& s. Sun (1995), ${ }^{d}$ Taylor \& McLennan (2009). ${ }^{e}$ Grevesse et al. (2007).

Table A2. Extended set of surface growth reactions for the growth of dust particles in addition to Table 1 in Helling et al. (2008b). The total sum of surface growth reaction taken into account here is 79 .

\begin{tabular}{|c|c|c|c|}
\hline Index $r$ & Solid $s$ & Surface reaction & Key species \\
\hline 61. & $\mathrm{MgO}[\mathrm{s}]$ & $2 \mathrm{MgH}+2 \mathrm{H}_{2} \mathrm{O} \longrightarrow 2 \mathrm{MgO}[\mathrm{s}]+3 \mathrm{H}_{2}$ & $\mathrm{MgH}$ \\
\hline 62. & $\mathrm{MgSiO}_{3}[\mathrm{~s}]$ & $2 \mathrm{MgH}+2 \mathrm{SiO}+4 \mathrm{H}_{2} \mathrm{O} \longrightarrow 2 \mathrm{MgSiO}_{3}[\mathrm{~s}]+5 \mathrm{H}_{2}$ & $\min \{\mathrm{MgH}, \mathrm{SiO}\}$ \\
\hline 63. & & $\mathrm{MgH}+\mathrm{SiH}+3 \mathrm{H}_{2} \mathrm{O} \longrightarrow \mathrm{MgSiO}_{3}[\mathrm{~s}]+4 \mathrm{H}_{2}$ & $\min \{\mathrm{MgH}, \mathrm{SiH}\}$ \\
\hline 64. & & $2 \mathrm{MgH}+2 \mathrm{SiN}+6 \mathrm{H}_{2} \mathrm{O} \longrightarrow 2 \mathrm{MgSiO}_{3}[\mathrm{~s}]+7 \mathrm{H}_{2}+\mathrm{N}_{2}$ & $\min \{\mathrm{MgH}, \mathrm{SiN}\}$ \\
\hline 65. & & $\mathrm{MgS}+\mathrm{Si}+3 \mathrm{H}_{2} \mathrm{O} \longrightarrow \mathrm{MgSiO}_{3}[\mathrm{~s}]+\mathrm{H}_{2} \mathrm{~S}+2 \mathrm{H}_{2}$ & $\min \{\mathrm{MgS}, \mathrm{Si}\}$ \\
\hline 66. & & $2 \mathrm{MgN}+2 \mathrm{Si}+3 \mathrm{H}_{2} \mathrm{O} \longrightarrow 2 \mathrm{MgSiO}_{3}[\mathrm{~s}]+3 \mathrm{H}_{2}+\mathrm{N}_{2}$ & $\min \{\mathrm{MgN}, \mathrm{Si}\}$ \\
\hline 67. & $\mathrm{Mg}_{2} \mathrm{SiO}_{4}[\mathrm{~s}]$ & $2 \mathrm{MgH}+\mathrm{SiO}+3 \mathrm{H}_{2} \mathrm{O} \longrightarrow \mathrm{Mg}_{2} \mathrm{SiO}_{4}[\mathrm{~s}]+4 \mathrm{H}_{2}$ & $\min \{2 \mathrm{MgH}, \mathrm{SiO}\}$ \\
\hline 68. & & $4 \mathrm{MgH}+2 \mathrm{SiH}+8 \mathrm{H}_{2} \mathrm{O} \longrightarrow 2 \mathrm{Mg}_{2} \mathrm{SiO}_{4}[\mathrm{~s}]+11 \mathrm{H}_{2}$ & $\min \{2 \mathrm{MgH}, \mathrm{SiH}\}$ \\
\hline 69. & & $4 \mathrm{MgH}+2 \mathrm{SiN}+8 \mathrm{H}_{2} \mathrm{O} \longrightarrow 2 \mathrm{Mg}_{2} \mathrm{SiO}_{4}[\mathrm{~s}]+\mathrm{N}_{2}+10 \mathrm{H}_{2}$ & $\min \{2 \mathrm{MgH}, \mathrm{SiN}\}$ \\
\hline 70. & & $2 \mathrm{MgS}+\mathrm{Si}+4 \mathrm{H}_{2} \mathrm{O} \longrightarrow \mathrm{Mg}_{2} \mathrm{SiO}_{4}[\mathrm{~s}]+2 \mathrm{H}_{2} \mathrm{~S}+2 \mathrm{H}_{2}$ & $\min \{2 \mathrm{MgS}, \mathrm{Si}\}$ \\
\hline 71. & & $2 \mathrm{MgN}+\mathrm{Si}+4 \mathrm{H}_{2} \mathrm{O} \longrightarrow 2 \mathrm{Mg}_{2} \mathrm{SiO}_{4}[\mathrm{~s}]+\mathrm{N}_{2}+4 \mathrm{H}_{2}$ & $\min \{2 \mathrm{MgN}, \mathrm{Si}\}$ \\
\hline 72. & $\mathrm{SiO}_{2}[\mathrm{~s}]$ & $2 \mathrm{SiH}+4 \mathrm{H}_{2} \mathrm{O} \longrightarrow 2 \mathrm{SiO}_{2}[\mathrm{~s}]+5 \mathrm{H}_{2}$ & $\mathrm{SiO}$ \\
\hline 73. & & $2 \mathrm{SiN}+4 \mathrm{H}_{2} \mathrm{O} \longrightarrow 2 \mathrm{SiO}_{2}[\mathrm{~s}]+\mathrm{N} 2+4 \mathrm{H}_{2}$ & $\mathrm{SiN}$ \\
\hline 74. & $\mathrm{SiO}[\mathrm{s}]$ & $2 \mathrm{SiH}+2 \mathrm{H}_{2} \mathrm{O} \longrightarrow 2 \mathrm{SiO}[\mathrm{s}]+3 \mathrm{H}_{2}$ & $\mathrm{SiO}$ \\
\hline 75. & & $2 \mathrm{SiN}+2 \mathrm{H}_{2} \mathrm{O} \longrightarrow 2 \mathrm{SiO}[\mathrm{s}]+\mathrm{N}_{2}+2 \mathrm{H}_{2}$ & $\mathrm{SiN}$ \\
\hline 76. & $\mathrm{Fe}[\mathrm{s}]$ & $2 \mathrm{FeH}+\mathrm{H}_{2} \longrightarrow 2 \mathrm{Fe}[\mathrm{s}]+2 \mathrm{H}_{2}$ & $\mathrm{FeH}$ \\
\hline 77. & $\mathrm{FeO}[\mathrm{s}]$ & $2 \mathrm{FeH}+2 \mathrm{H}_{2} \mathrm{O} \longrightarrow 2 \mathrm{FeO}[\mathrm{s}]+3 \mathrm{H}_{2}$ & $\mathrm{FeH}$ \\
\hline 78. & $\mathrm{FeS}[\mathrm{s}]$ & $2 \mathrm{FeH}+2 \mathrm{H}_{2} \mathrm{O} \longrightarrow 2 \mathrm{FeS}[\mathrm{s}]+3 \mathrm{H}_{2}$ & $\mathrm{FeH}$ \\
\hline 79. & $\mathrm{Fe}_{2} \mathrm{O}_{3}[\mathrm{~s}]$ & $2 \mathrm{FeH}+3 \mathrm{H}_{2} \mathrm{O} \longrightarrow \mathrm{Fe}_{2} \mathrm{O}_{3}[\mathrm{~s}]+4 \mathrm{H}_{2}$ & $\mathrm{FeH}$ \\
\hline
\end{tabular}

